Florida International University FIU Digital Commons

6-29-2018

\title{
The Oscillatory Shear Index: Quantifications for Valve Tissue Engineering and a Novel Interpretation for Calcification
}

Alex Williams

awill234@fiu.edu

DOI: $10.25148 /$ etd.FIDC006850

Follow this and additional works at: https://digitalcommons.fiu.edu/etd

Part of the Molecular, Cellular, and Tissue Engineering Commons

\section{Recommended Citation}

Williams, Alex, "The Oscillatory Shear Index: Quantifications for Valve Tissue Engineering and a Novel Interpretation for Calcification" (2018). FIU Electronic Theses and Dissertations. 3784.

https://digitalcommons.fiu.edu/etd/3784 


\title{
FLORIDA INTERNATIONAL UNIVERSITY
}

Miami, Florida

THE OSCILLATORY SHEAR INDEX: QUANTIFICATIONS FOR VALVE TISSUE ENGINEERING AND A NOVEL INTERPRETATION FOR CALCIFICATION

\author{
A thesis submitted in partial fulfillment of \\ the requirements for the degree of \\ MASTER OF SCIENCE \\ in \\ BIOMEDICAL ENGINEERING \\ by \\ Alexander T. Williams
}


To: Dean John Volakis

College of Engineering and computing

This thesis, written by Alexander T. Williams and entitled The Oscillatory Shear Index: Quantifications for Valve Tissue Engineering and a Novel Interpretation for Calcification, having been approved in respect to style and intellectual content, is referred to you for judgment.

We have read this thesis and recommend that it be approved.

$\begin{array}{r}\text { Joshua Hutcheson } \\ \text { Mike Sukop } \\ \hline\end{array}$

Sharan Ramaswamy, Major Professor

Date of Defense: June 29, 2018

The thesis of Alexander T. Williams is approved.

Dean John Volakis

College of Engineering and Computing

Andrés G. Gil

Vice President for Research and Economic Development and Dean of the University Graduate School

Florida International University, 2018 


\section{ACKNOWLEDGMENTS}

I wish to thank my advisor Dr. Sharan Ramaswamy for his continuous guidance. Also, my committee members Dr. Joshua Hutcheson and Dr. Mike Sukop for guiding my research in the right direction and my lab mates for their continued support. 


\title{
ABSTRACT OF THE THESIS \\ THE OSCILLATORY SHEAR INDEX: QUANTIFICATIONS FOR VALVE TISSUE ENGINEERING AND A NOVEL INTERPRETATION FOR CALCIFICATION
}

by

\author{
Alexander T. Williams \\ Florida International University, 2018 \\ Miami, Florida \\ Professor Sharan Ramaswamy, Major Professor
}

Heart valve tissue engineering (HVTE) stands as a potential intervention that could reduce the prevalence of congenital heart valve disease in juvenile patients. Prior studies in our laboratory have utilized mechanobiological testing to quantify the forces involved in the development of heart valve tissue, utilizing a Flow-Stretch-Flexure (FSF) bioreactor to condition bone marrow stem cells (BMSCs)-derived valve tissue. Simulations have demonstrated that certain sets of flow conditions can introduce specific levels of oscillatory shear stress (OSS)-induced stimuli, augmenting the growth of engineered valves as well as influencing collagen formation, extracellular matrix (ECM) composition and gene expression. The computational findings discussed in this thesis outline the methods in which flow conditions, when physiologically relevant, induce specific oscillatory shear stresses which could not only lead to an optimized valve tissue phenotype (at $0.18 \leq \mathrm{OSI} \leq 0.23$ ), but could identify native valve tissue remodeling indicative of aortic valve disease. 


\section{TABLE OF CONTENTS}

CHAPTER

PAGE

1. INTRODUCTION

1.1. Problem statement

1.2. Current Barriers

2

1.3. Specific Aims

4

1.4. Overview of proposed methodologies

4

2. LITERATURE REVIEW 6

2.1. Heart Valve Disease 6

2.2. Heart valve Replacement and Repair procedures $\quad 7$

$\begin{array}{ll}2.2 .1 \text {. Replacement } & 7\end{array}$

2.2.2. Repair 8

2.3 Tissue Engineering and Regenerative approaches for

Heart Valves 8

2.3.1 Motivation $\quad 8$

$\begin{array}{ll}\text { 2.3.2 Challenges } & 9\end{array}$

2.3.3 Literature review of the field 9

2.4 Computational Fluid Dynamics (CFD) and Heart Valve Tissue

$\begin{array}{ll}\text { Engineering } & 12\end{array}$

$\begin{array}{ll}\text { 2.4.1 CFD and heart valves } & 12\end{array}$

2.4.2 Previous Studies in Our Laboratories $\quad 12$

2.4.2.1. Flow systems $\quad 12$

2.4.2.1.1 Flow-Stretch-Flexure Bioreactor $\quad 12$

2.4.2.1.2 Shear Stress Cell Assay System (Bioflux) 13

2.4.2.2 Steady Flows $\quad 13$

2.4.2.3 Combined Flexure and Steady Flow (Flex-Flow) 13

2.4.2.4 Non-Physiological Pulsatile Flow (NPPF) 14

3. SPECIFIC AIM 1 15

3.1. Methodology for Specific Aim $1 \quad 16$

$\begin{array}{ll}\text { 3.1.1. Inlet and Outlet Boundary Conditions } & 17\end{array}$

3.1.1.1. Pulmonary Arterial Waveform $\quad 17$

3.1.1.2. Aortic Waveform 18

3.1.1.3. Superior Vena Caval Waveform 18

$\begin{array}{lr}\text { 3.1.2. Numerical Independence } & 19\end{array}$

3.1.2.1. Mesh Independence 19

3.1.2.2. Timestep Independence 19

3.1.2.3. Cyclic Independence 20

$\begin{array}{ll}\text { 3.1.3. CFD Validation } & 21\end{array}$

3.1.4. Mechanobiological Testing 21

3.1.5. Gene Expression Analysis 22

3.1.6. Statistical Analysis 23 
3.2. Results of Specific Aim $1 \quad 23$

3.2.1. Velocity Streamlines 23

3.2.1.1. Pulmonary Artery 24

3.2.1.2. Aorta $\quad 25$

3.2.1.3. Superior Vena Cava 26

3.2.2. Vorticity Plots 26

3.2.2.1. Pulmonary Artery 27

3.2.2.2. Aorta 28

3.2.2.3. Superior Vena Cava $\quad 29$

3.2.3. Instantaneous Wall Shear Stress Approximations 29

3.2.3.1. Pulmonary Artery $\quad 30$

3.2.3.2. Aorta 31

3.2.3.3. Superior Vena Cava $\quad 32$

3.2.4. Oscillatory Shear Index 33

3.2.5. Gene Expression Results 34

3.3. Discussion of Specific Aim 1

4. $\quad$ SPECIFIC AIM $2 \quad 42$

4.1. Methodology for Specific Aim 2

4.1.1. Inlet and Outlet Boundary Conditions 46

4.1.2. Numerical Independence 46

4.1.2.1. Mesh Independence 46

4.1.2.2. CFL-timestep selection and subsequent

4.1.2.3. Cyclic Independence $\quad 47$

4.2. Results of Specific Aim 2

4.2.1. Time-Averaged Shear Stress $\quad 47$

4.2.1.1. Healthy Valve Model 47

4.2.1.2. Diseased Valve Model 49

4.2.2. Oscillatory Shear Index $\quad 50$

4.2.2.1. Healthy Valve Model $\quad 50$

4.2.2.2. Diseased Valve Model $\quad 50$

4.2.2.3. Regions that developed calcification $\quad 51$

4.2.3. \% Change in OSI 51

4.3. Discussion of Specific Aim 2

5. CONCLUSION 55

$\begin{array}{ll}\text { REFERENCES } & 56\end{array}$ 
Table 1: Quantitative real-time polymerase chain reaction primer sequences for gene expression analysis of HBMSCs......................... 22

Table 2: Healthy and diseased OSI on both ventricularis and fibrosa sides of valve with percent of calcified plaque occupying each cusp.

Particularly, there was a significant change in OSI between the diseased and healthy LCC $(\mathrm{p}<0.05)$.

Table 3: OSI on cusp-designated areas of valve fibrosa (A1-A4) for each region. Note that $\mathrm{A} 3$ and $\mathrm{A} 4$ are the calcified regions and unaffected regions of the calcified valve, respectively, while $\mathrm{A} 1$ and $\mathrm{A} 2$ are the corresponding calcified regions and corresponding unaffected regions of the healthy valve Also note that R1, R2 and R3 represented regions where calcification had occurred (R1: across NCC, R2: across RCC, R3: across LCC). Mean \pm SEM is provided for each area. A slight shift in OSI (1.6\%) occurred

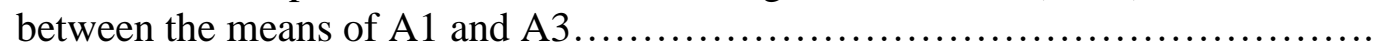

Table 4: Percent (\%) change in mean OSI of cusp-designated regions and in mean OSI across distinct valvular cusps. There was a markedly low percent change between the means of $\mathrm{A} 1$ and $\mathrm{A} 3(1.6 \%)$ compared to the change between the means of A2 and A4 (36\%). 
Fig. 1: (a) FSF Bioreactor Chamber schematic [25] used to design (b) a simulated bioreactor model modelled on ANSYS $®$ CFX ............................... 2

Fig. 2: Pulmonary arterial waveform with peak velocity at $0.095 \mathrm{~m} / \mathrm{s}$ and a mean velocity at $0.022 \mathrm{~m} / \mathrm{s}$.

Fig. 3: Aortic waveform with peak velocity at $0.109 \mathrm{~m} / \mathrm{s}$ and a mean velocity at $0.0241 \mathrm{~m} / \mathrm{s}$

Fig. 4: Superior vena caval waveform with peak velocity at $0.025 \mathrm{~m} / \mathrm{s}$ and a mean velocity at $0.81 \mathrm{~m} / \mathrm{s}$.

Fig. 5: The FSF bioreactor quadrilateral mesh as shown from both the Xz-axis (a) and the yz-axis (b). The mesh density of the geometry was optimized at $4.72 \times 10^{5}$ nodes and $4.41 \times 10^{5}$ elements with the mesh refined around the samples of the bioreactor, as depicted from the xz-axis (c) and the xy-axis (d) with the black arrow pointing to the proximal wall of each respective sample and the purple arrow pointing to the distal wall

Fig. 6: Velocity waveforms (a) which produced corresponding shear stress profiles (b) to obtain a (i) physiologically relevant OSI (OSI $=0.23$ )

(ii) low-OSI (OSI = 0.10) and (iii) high-OSI (OSI $=0.38)$

Fig. 7: Instantaneous velocity contours of the pulmonary arterial profile under five equitemporal time points from both the top plane (proximal walls) and the bottom plane (distal walls). Peak velocity is distinguished at the peak of the waveform at $0.16 \mathrm{~s}$.

Fig. 8: Instantaneous velocity contours of the aortic profile under five equitemporal time points from both the top plane (proximal walls) and the bottom plane (distal walls). Peak velocity is distinguished at the peak of the waveform at $0.16 \mathrm{~s}$.

Fig. 9: Instantaneous velocity contours of the superior vena caval profile under five equitemporal time points from both the top plane (proximal walls) and the bottom plane (distal walls). Peak velocity is distinguished at the peak of the waveform at $0.20 \mathrm{~s}$.

Fig. 10: Sample-intersecting vorticity contour maps of the pulmonary arterial profile at seven timepoints.

Fig. 11: Sample-intersecting vorticity contour maps of the aortic profile at 
Fig. 12: Sample-intersecting vorticity contour maps of the superior vena caval profile at seven timepoints.

Fig. 13: Instantaneous sample shear stress contours for pulmonary arterial simulation. For time points $0.00 \mathrm{~s}, 0.04 \mathrm{~s}, 0.08 \mathrm{~s}, 0.16 \mathrm{~s}, 0.24 \mathrm{~s}, 0.28 \mathrm{~s}$ and $0.32 \mathrm{~s}$, the shear stress averages were $0.08 \pm 0.002,0.46 \pm 0.009,1.8 \pm 0.02$, $2.5 \pm 0.02,1.3 \pm 0.01,0.76 \pm 0.009$ and $0.28 \pm 0.007$, respectively.

Fig. 14: Instantaneous sample shear stress contours for aortic simulation. For time points $0.00 \mathrm{~s}, 0.04 \mathrm{~s}, 0.08 \mathrm{~s}, 0.16 \mathrm{~s}, 0.24 \mathrm{~s}, 0.28 \mathrm{~s}$ and $0.32 \mathrm{~s}$, the shear stress averages were $0.21 \pm 0.003,0.59 \pm 0.006,2.0 \pm 0.02$, $2.7 \pm 0.02,1.2 \pm 0.01,0.49 \pm 0.009$, and $-0.06 \pm 0.008$, respectively

Fig. 15: Instantaneous sample shear stress contours for superior vena cava simulation. For time points $0.04 \mathrm{~s}, 0.08 \mathrm{~s}, 0.12 \mathrm{~s}, 0.20 \mathrm{~s}$, $0.28 \mathrm{~s}, 0.32 \mathrm{~s}$, and $0.36 \mathrm{~s}$, the shear stress averages were $0.024 \pm 0.0003$, $0.043 \pm 0.0004,0.057 \pm 0.0004,0.047 \pm 0.0003,0.017 \pm 0.0002$, $0.004 \pm 0.0002$, and $-0.003 \pm 0.0002$ respectively $\ldots$

Fig. 16: OSI contours $(n=3)$ of the samples housed within the FSF bioreactor. The mean OSI of the distal walls for the pulmonary arterial, aortic, superior vena cava, square wave and Flex-Flow conditions were $0.28 \pm 0.002$, $0.25 \pm 0.002,0.27 \pm 0.001,0.45 \pm 0.023$, and $0.117 \pm 0.003$, respectively.

On the other hand, the corresponding mean OSI for the proximal wall were as follows: $0.18 \pm 0.002,0.10 \pm 0.002,0.11 \pm 0.001,0.29 \pm 0.019$, and $0.094 \pm 0.10$.

Fig. 17: Gene expression following 48 hours of HBMSCs exposed to varying OSI levels. (a) A statistically significant ( $\mathrm{p}<0.05)$ expression of CD31, $\alpha$-SMA was found in the physiologically relevant OSI-treated group as compared to the low and high OSI counterparts. (b) A significantly higher expression $(\mathrm{p}<0.05)$ of BMP2 was also observed in the physiologically relevant OSI groups as compared to the other two groups. No significant differences $(p>0.05)$ were found to occur in the expression of osteocalcin between the groups. (c) KLF2A and NOTCH1 genes exhibited a significantly higher expression $(\mathrm{p}<0.05)$ in the physiologically relevant OSI group. Finally, TGFb1 was significantly down-regulated in the low OSI and physiologically relevant groups $(\mathrm{p}<0.05)$ in comparison to high OSI-treatment wherein it was upregulated.

Fig. 18: (a) Healthy aortic valve Fibrosa geometry in early diastole, with left coronary cusp (LCC), right coronary cusp (RCC) and non-coronary cusp (NCC), before and (b) after calcification. (c)The simulated healthy 
valve fibrosa model and (d) its corresponding mesh, had calcified nodules (black) added across the leaflets for (e) the diseased valve model and (f) its corresponding mesh. Areas of interest (A1, A2, A3, A4) on those regions represented both calcified areas (A3) and unaffected areas of the diseased valve (A4) and the areas corresponding to the calcified regions (A1) and unaffected regions (A2) on the healthy valve. (h) On the diseased valve, calcified deposits were analyzed both independently and as a collective zone (area A3, black) [A3-NCC $\left(126 \mathrm{~mm}^{2}\right.$, located at distal belly of NCC), A3-RCC (117.2 $\mathrm{mm}^{2}$, located at tips and proximal belly of RCC) and A3-LCC (51.9 $\mathrm{mm}^{2}$, located at proximal belly of LCC)] along with the regions surrounding the deposits (area A4, red)[A4-NCC: $194.3 \mathrm{~mm}^{2}$; A4-RCC: $203.6 \mathrm{~mm}^{2}$; A4-LCC: $240 \mathrm{~mm}^{2}$ ] within each specific cusp.

(g) The deposit regions corresponding to the diseased valve were then analyzed across the healthy valve (area A1, black) [A1-NCC: $144.93 \mathrm{~mm}^{2}$; A1-RCC: $81.08 \mathrm{~mm}^{2}$; A1-LCC:17.07 $\mathrm{mm}^{2}$ ] along with the areas consistent with the regions surrounding the deposits (area A2, red) [A2-NCC: $151.26 \mathrm{~mm}^{2}$; A2-RCC: $218.16 \mathrm{~mm}^{2}$; A2-LCC:291.24 $\mathrm{mm}^{2}$ ]

Fig. 19: Healthy fibrosa geometry plots of (a) axial time-averaged wall shear stress (TAWSS) (mean: $0.091 \mathrm{dynes} / \mathrm{cm}^{2}$ ) and (b) OSI (mean: 0.0998) as well as diseased fibrosa geometry plots for (c) axial TAWSS (mean: 0.323 dynes/cm2) and (d) OSI (mean: 0.1557).

Fig. 20: Healthy ventricularis geometry plots of (a) axial time-averaged wall shear stress (TAWSS) (mean: 1.25 dynes $/ \mathrm{cm}^{2}$ ) and (b) OSI (mean: 0.003457) as well as diseased ventricularis geometry plots for (c) axial TAWSS (mean: 1.95 dynes $/ \mathrm{cm}^{2}$ ) and (d) OSI (mean: 0.01). 49 


\section{LIST OF EQUATIONS}

EQUATION

PAGE

Eq.1: Oscillatory Shear Index.............................................. 3

Eq.2: The Navier Stokes equation represented by the momentum equation (a) and the continuity equation (b) ........................ 12 


\section{ABBREVIATIONS AND ACRONYMS}

\begin{tabular}{|c|c|}
\hline AVS & Aortic valve stenosis \\
\hline $\mathrm{BHV}$ & Bioprosthetic Heart Valve Heart Valve \\
\hline BMSC & Bone marrow stem cell \\
\hline CFD & Computational Fluid Dynamics \\
\hline $\mathrm{ECM}$ & Extracellular Matrix \\
\hline EHV & Engineered Heart Valve \\
\hline $\mathrm{LCC}$ & Left Coronary Cusp \\
\hline MHV & Mechanical Heart Valve Heart Valve \\
\hline NCC & Non-Coronary Cusp \\
\hline NPPF & Non-Physiological Pulsatile Flow \\
\hline OSI & Oscillatory Shear Index \\
\hline OSS & Oscillatory Shear Stress \\
\hline $\mathrm{RCC}$ & Right Coronary Cusp \\
\hline SS & Steady State \\
\hline TAWSS & Time-Average Wall Shear Stresses \\
\hline TEHV & Tissue Engineered Heart Valve \\
\hline VEC & Valvular Endothelial Cell \\
\hline
\end{tabular}


Wall Shear Stress 


\section{INTRODUCTION}

\subsection{Problem Statement:}

Heart valve disease remains a prevalent concern in patients with cardiovascular disease and pediatric patients with congenital heart defects. Several potential interventions can be applied to these patients to mitigate the effects of this condition, one of which involves the application of engineered heart valves (EHVs) [1,2]. For this modality, the environment necessary for the appropriate development of engineered valve tissue would require a specific set of flow conditions to be met. These flow condition regimes have the potential to induce appreciable development and gene expression of heart valve tissue [4, 2, 3, 5], with physiological flow conditions speculated to significantly enhance EHV phenotype. Recapitulating the biological circumstances necessary for conditioning EHVs would then require an understanding of the mechanobiological forces crucial to EHV fabrication. Genes, such as klf2a and cd31, induce favorable maturation in vascular endothelial cells (VECs), which aid in the activation of regenerative processes of heart valve tissue. These genes also induce viability in bone marrow stem cells (BMSCs), which were found to be imperative in the fabrication of engineered valves. Such genes were found to be present when certain flow parameters, such as shear stress, occurred at the tissue level $[6,7]$.

These flow patterns have also been shown to influence valve blood flow mechanics in native valve tissue. From a hemodynamical standpoint, abnormal flow physics have been shown to progress aortic valve disease by influencing the accumulation of calcified plaque deposits along valve leaflets $[9,8,2,10]$. Further studies have shown that aortic valve remodeling is affected by an abnormal flow regime, such as was found with wall 
shear stress, which could be an initiator in the process of valve calcification [11]. Based on these implications, we hypothesize that the oscillatory flow physics that exist at the tissue level would not only indicate gene expression that corresponds to a potential optimization in EHV formation and phenotype, but could also act as an indicator for the remodeling of the tri-leaflet valve systems in terms of valve calcification.

\subsection{Current Barriers:}

Extensive research in the fields of biomechanics realize EHV development across a gene-tissue hierarchy. In our lab, the research carried out has implemented a simulated Flow-Stretch-Flexure (FSF) bioreactor (Fig. 1) to identify the conditions that moderate the fluid flow parameters involved in engineering valve tissue. Computational studies have shown that there is a correlation between oscillatory shear stresses and valve development, including appreciable collagen formation

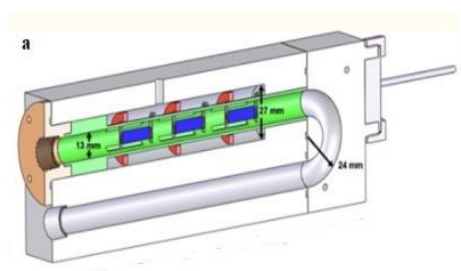

Fig.1: (a) FSF Bioreactor Chamber schematic [25] used to design (b) a simulated bioreactor model modelled on ANSYS ${ }^{\circledR}$ CFX

and valvular phenotype $[5,12,13]$. These findings suggest that there should then be a particular range of oscillatory shear stresses (OSS) that could correspond not only to engineered valve tissue augmentation, but also to the expression of genes that sustain the architecture and functionality of EHVs. However, the distinct conditions that would express these levels of OSS have not yet been determined. Furthermore, there is little evidence on whether such conditions would impose similar levels of OSS across an appropriate physiologically relevant model, i.e. healthy aortic valve leaflets, or if such 
conditions derive such OSS levels specifically across the geometric configuration of the poly(l-lactic acid) / poly(l-glutamic acid) (PLLA/PLGA) scaffold-based FSF bioreactor environment.

Retrospectively, since fluid flow is crucial in the facilitation of OSS [14, 6], a previous report [12] suggests that a physiologically relevant, vessel-specific flow regime could produce a range of OSS, summarized as an oscillatory shear index (OSI) between 0 and 0.5 (Eq. 1), that is theorized to optimize valvular phenotypic characteristics and gene expression specific to EHVs [12]. Considering the ramifications of this study, the FSF bioreactor geometry was redeployed to simulate physiological conditions for identifying flow parameters across a typical bioreactor environment.

$$
O S I=0.5 \cdot\left(1-\frac{\left|\int_{0}^{T} W S S d t\right|}{\int_{0}^{T}|W S S| d t}\right)
$$

Equation 1: Oscillatory Shear Index

There are several flow parameters that could influence the development of valve disease in native tri-leaflet valves $[9,2,15,16,1718,10]$. However, few studies have looked at how the remodeling of the valvular ECM environment could be affected by OSS-induced stimuli and whether this remodeling could correlate to adverse effects, i.e. calcified plaque deposits, which lead to valve disease. Subsequently, physiological conditions were prescribed to a healthy heart valve conduit, undergoing mid-diastole, to correlate the flow parameters along the scaffolds to those involved with the geometry of the valve leaflets when exposed to similar conditions. Additional simulations were run, 
adding calcified deposits to the heart valve geometry to identify if there were any notable variations in the OSI distribution of a diseased valve model geometry.

\subsection{Specific Aims:}

The hypothesis was tested using the following specific aims:

Specific Aim 1: Simulating physiologically relevant flow conditions across a 3Dcomputational tissue-level environment, and demarcating flow physics across the BMSC-layered scaffolds of the model.

Specific Aim 2: Modelling physiologically relevant flow conditions across heart valve models, both healthy and calcified, and distinguishing the flow parameters specific to both normal valve hemodynamics and region-defined valve calcification.

\subsection{Methodologies:}

To efficiently carry out and run the simulated experiments, an Intel Xeon® X5550 central processing unit with dual processors (2.66 and $2.667 \mathrm{GHz}$ ) was employed due to its processing power rendering it capable of maintaining multiple simulations. The study also used a Windows ${ }^{\circledR} 7$ 64-bit operating system which met with the software requirements necessary from the appropriate simulation software and the graphics that coincide with the results.

The tissue-based simulations, executed through ANSYS Workbench®2016 (ANSYS Inc., Canonsburg, PA) used a set of physiologically relevant, vessel-specific velocity profiles (fig. 2, 3, 4) prescribed to the inlet of the given geometry. The tissue-based simulations used the fluid parameters of cell culture basal media, in which the simulated fluid was Newtonian, produced laminar flow, and had both a density of $1.01 \mathrm{~g} / \mathrm{cm} 3$ and 
a viscosity of $1.27 \mathrm{cP}$. For each simulation run, transient data needed to reach an appropriate level of accuracy during the solution phase of each computation, which required the convergence criteria to be set to $1 \times 10^{-9}$ and each given time step, of the respective timeframe for each profile, to go through 50 iterations to ensure result precision. Computational properties of tissue-level studies, such as the geometry or the fluid properties, were validated with previous simulated pulsatile studies.

For the valve-based computational geometries, 3D reconstructions of both a healthy valve-aortic root (AVAR) anatomy and a diseased AVAR anatomy were modelled within SolidWorks ${ }^{\circledR 2015 a}$. Each model was then separately imported into ANSYS Workbench®2016, similar to the tissue-based simulations, using the aortic-specific velocity profile (fig. 3) as an inlet boundary condition. The simulations used the fluid parameters of blood, a non-Newtonian simulated fluid with a density of $1.06 \mathrm{~g} / \mathrm{cm} 3$ and a viscosity of $3.5 \mathrm{cP}$. The boundary conditions were employed into the model with an $80 \mathrm{~mm} \mathrm{Hg}$-pressure profile prescribed to the outlet and no-slip boundary conditions applied to the overall structure of the model. The valve-based studies were validated by computing a $120 \mathrm{~mm} \mathrm{Hg}$ pressure reading at the inlet when utilizing pulsatile conditions across the healthy valve.

After collecting OSI and time-averaged wall shear stress (TAWSS) data from the tissue-based simulations, the quantifications were compared to data originating from shear stress boundary conditions used for shear stress experiments, which were executed within a Bioflux 1000 system (Fluxion Biosciences, South San Francisco, CA) for BMSCs. Associations between the given parameters were able to determine whether 
there was a link between the acquired computed data and the genes expressed during the shear stress experiments.

For the flow quantifications found within this study, the mean and standard error of the mean for both cases were given for each parameter used. Statistical differences between the simulated data and the experimental data were confirmed using ANOVA tests $(\mathrm{p}<0.05)$

\section{LITERATURE REVIEW}

\subsection{Heart Valve Disease}

Heart valve disease has been increasing in incidence in the United States with an estimated 1.5 million citizens affected [19]. Some of the symptoms of heart valve disease include regurgitation, which involves blood flowing back into the ventricle during diastole, and stenosis, which involve occlusions of the valve orifice most prominently from the stiffening and thickening of the valves, which can be due to calcification. The most common cases of aortic valve stenosis (AVS) occur as calcific aortic stenoses, which can be developed with age, and congenital bicuspid aortic stenoses, diagnosed at birth $[15,18]$.

AVS, once considered an age-related degenerative condition resulting in the formation of calcium deposits in heart valves, has recently been shown to be involved in an intricate set of processes similar to atherosclerosis. These processes have been demonstrated to damage the valvular endothelial layer due to an increase in mechanical stresses, thought to be an initializing event for developing AVS [20, 18]. The flow of blood through the aortic valve accounts for these fluid-induced mechanical forces, such 
as the shear stress across the valves, leading to abnormalities within the cellular architecture of both the fibrotic and ventricularis sides of aortic valves, given any quantifiable deviations of these fluid-induced forces.

VECs, undergoing physiological shear stress values, respond by expressing antiinflammatory genes; however, when they are exposed to abnormal fluid flow shear stress, inflammatory mediators were activated. Studies have also shown the activation of calcification-related genes after VECs have been exposed to abnormal shear stresses. $[11,21]$. Though it has been shown that discrepancies in mechanotransductive forces and tissue hemodynamics could lead to endothelial damage, these flow field mechanics, given normal, healthy circumstances, substantially maintain the environment of engineered valve tissue $[2,10,18-21]$.

\subsection{Heart Valve Replacement and Repair Procedures}

\subsection{1. $\underline{\text { Replacement }}$}

Heart valve prosthetics have been recognized as a suitable method to replace diseased heart valves. Early heart valve prosthetics during early valve prosthetic implantation used mechanical heart valves (MHVs). MHVs used in clinical trials had several different variants, including the ball-and-cage mechanical valve, the rotating disk valve and bileaflet trileaflet mechanical valves. However, MHVs were associated with irregular hemodynamics, such as high velocity backflow jets and high shear stresses across hinges of the valves, causing hemolytic dysfunction, and requiring patients to take life-long anticoagulation medications. [3] 
In such cases, bioprosthetic heart valves (BHVs) were developed to better acclimate with the environment of the aorta due to its integration of native tissue from animals (xenografts), human donors (homografts), or from the patient's body (autografts). The main advantage of bioprosthetic heart valves was that patients would not be required to take life-long anticoagulants. However, the risk for BHV replacements came from the structural valve deterioration that occurred from the narrow durability and stability of BHVs, which would then increase the chance for reoperation, particularly for pediatric patients [3, 23].

\subsubsection{Repair}

Valvular surgery is the other intervention which could be used to treat damaged, diseased or calcified heart valves, typically involving open heart surgery. Several techniques have been developed for the repair of aortic valves, including the Ross procedure, which involves aortic repair utilizing pulmonary valve tissue, and minimal access aortic valve surgery through which sutureless valves could potentially improve. It is the more ideal choice for severe regurgitation due to valve prolapse, though aortic root remodeling could also ultimately be necessary for this type of repair. Foregoing valve replacement with repair could sometimes lead to a postoperative revision, though the reoperation rate usually occurs between $2-3 \%$, not looking beyond the results that occur 10 years post-operation. [24]

\subsection{Tissue Engineering and Regenerative Methods for Heart Valves}

\subsubsection{Motivation}

An appropriate potential alternative for replacement of both mechanical and bioprosthetic heart valves would involve the use of regenerative approaches to construct 
tissue engineered heart valves (TEHVs). TEHVs are also associated with a more native set of hemodynamics in comparison to mechanical valves, while also having a lower potential for reoperation in comparison to bioprosthetic heart valves. Additionally, unlike both valve replacement modalities, TEHVs not only have a more natural blood flow regime due to their natural structure, but they have the capability to grow with the patient, making them suitable for pediatric patients $[4,1,25]$.

\subsubsection{Challenges}

There are several pitfalls that need to be addressed involving the construction of TEHVs, and consequently restricting their use within the clinic. TEHVs, though biocompatible, have been shown to undergo complications at the cellular level, in which interstitial and endothelial cells have limited communication between the extracellular matrix. Additionally, TEHVs are also subject to flow physics used for conditioning in controlled environments (i.e., bioreactors) that can vary to the physiological blood flow physics associated with native valves $[4,3]$. Particularly with decellularized TEHVs, there is also the threat of valvular deterioration that could be caused from inflammatory rejection [4].

\subsubsection{Literature Review of the Field}

There have been several approaches taken for the scaffolding and succeeding development of TEHVs, such as in the study by Konertz et al .[26], which involved implanting a decellularized porcine aortic valve xenograft into several patients (age median: 46 years) throughout a two-year timeframe, with the typical recellularization timespan ranging within four months. The results of the study revealed that the valves 
demonstrated similar characteristics to native healthy valves, particularly due to sustaining low, consistent transvalvular gradients [26].

Zhou et al. [27] performed polyethylene glycol (PEG)-ylation of decellularized aortic porcine valves. Mechanical testing revealed that there was a significant increase in the tensile strength of the aortic valve tissue. The study also looked into using branched PEG to facilitate the conjugation of bioactive agents, such as vascular endothelial growth factor (VEGF), onto the aortic valve. Human umbilical vein endothelial cells (HUVECs) were found to grow appreciably on the tissue when conjugated with VEGF and glycine-arginine-glycine-aspartate-serine-proline-cysteine (GRGDSPC) peptides after PEG-ylation.

Schmidt et al. [31] developed a method for minimally invasive surgery of TEHVs using bioreactor technology and adult stem cells. The trileaflet valves were integrated onto self-expanding stents and implanted into sheep. In vivo functionality and composition analyses revealed newly formed valve tissue with the extracellular matrix, producing higher levels of collagen and glycosaminoglycans than typically found in native tissue. A similar study was done by Weber et al [28], in which marrow stromalcell-based, autologous TEHVs were fabricated. Then, using Nitinol-based stents, these TEHVs were implanted into nonhuman primates as pulmonary valves, with composition and biomechanical analyses demonstrating that the biodegradable scaffold matrix was being replaced with endothelialized, layered, living tissue.

Syedain et al. [29] applied an off-the-shelf engineered valve within sheep, which resulted in endothelialization, colonization of interstitial-like cells within the engineered matrix, new extracellular deposits, such as elastin, retained functionality, and increased 
tissue remodeling 24 weeks after implantation with no calcification. The structural integrity was also deemed satisfactory according to the number of fatigue cycles the valve overcame within the time span (17 million cycles).

Engelmeyer et al.[14] created a Flow-Stretch-Flexure (FSF) bioreactor using mechanical stretching and bending of rectangular, nonwoven 50:50 blend poly (glycolic acid) (PGA) and poly(l-lactic acid) (PLLA) scaffolds to condition and optimize the development of bone marrow-derived mesenchymal stem cell (BMSC)-derived TEHVs. Using two mechanical stimuli physiologically relevant to heart valves, the cyclic flexure rate of the samples coupled with a laminar flow regime [Flex-Flow], the results of this study reported high levels of tissue formation, collagen content and effective stiffness similar to that of smooth muscle cell (SMC)-seeded scaffolds.

Ramaswamy et al [25] subsequently tested the validity of using physiological flow, stretch and flexure by developing a novel FSF bioreactor for BMSC-derived TEHVs. As opposed to the study, which looked at the effects of Flex-Flow conditions in optimizing engineered valve growth, the study by Ramaswamy et al. utilized physiologically relevant shear stress ranges (5-6 dynes/ $\left.\mathrm{cm}^{2}\right)$, leading to higher levels of collagen content compared to the levels found in Engelmeyer et al., which induced lower, subphysiological shear stress ranges. Furthermore, computational analyses done by Salinas et al. [6] and Salinas and Ramaswamy [6] identified the flow-mediated mechanics that took place within the confines of an FSF bioreactor environment. Specifically, fluidinduced oscillatory shear stresses were found to be substantial in the conditioning of TEHVs. 


\subsection{Computational Fluid Dynamics (CFD) and Heart Valve Tissue Engineering}

\subsubsection{CFD and Heart Valves}

Tissue-based computational analysis, employing the Navier-

Stokes Equations for continuity and momentum (Eq. 2), has been

a. $\rho\left(\frac{\partial u}{\partial x}+u \cdot \nabla u\right)=-\nabla \cdot P+\rho g+\mu \cdot \nabla^{2} u$

b. $\nabla \rho=0$

Equation 2: The Navier Stokes equation represented by the momentum equation (a) and the continuity equation (b)

utilized in studies involved with determining the flow physics and mechanical forces that occur across heart valves. Such analyses have also been applied within other domains in which valvular tissue is grown and conditioned (i.e., bioreactors). Previous computational studies have shown that native hemodynamics have certain levels of shear stresses that are imposed on aortic valve tissue, particularly at a range of 0 to 3.49 dynes $/ \mathrm{cm}^{2}[30]$.

\subsubsection{Previous Studies in Our Laboratories}

\subsubsection{Flow systems}

\subsection{Flow-Stretch-Flexure Bioreactor}

We have utilized an FSF bioreactor, which seeds human bone marrow stem cells across scaffolds that undergo certain degrees of flexure and bending, along with supplying a flow regime across the bioreactor chambers, to provide the mechanical forces necessary to sustain and even enhance the development of heart valve tissue. With a need to understand and control the flow physics that impart these mechanical forces across scaffolds to optimize the quality, phenotype, and growth of engineered heart valves, employing computational modelling and analysis of the flow conditions, including ANSYS Workbench ®2016 and Tecplot 360 EX ®2016, provide a sufficient segue to 
resolve this matter. In regard to the mechanical forces that are analyzed within these studies, principally, we have found that OSS, which oscillate due to the fluctuating of flow back and forth, can supplement the development of valvular tissue. Our laboratory has looked at the different sets of flow conditions within the bioreactor geometry, retaining certain values of oscillatory shear stresses, summarized as OSI, which range from 0 , indicating no oscillating shear patterns, to 0.5 , indicating high levels of oscillatory shear stresses.

\subsection{Shear Stress Cell Assay System (Bioflux)}

Studies within our lab have used the Bioflux 200 system to condition individual cells, or groups of cells, by using shear stress profiles to recapitulate multiple conditions before analyzing the cells' behavior, gene expression patterns, and morphology. Shear stress plates previously used have had an applicable shear stress range of 0-20 dynes $/ \mathrm{cm}^{2}$.

\subsubsection{Steady Flow}

Steady-state flow conditions set different cases where the scaffolds were flexed at varying conformations. When utilized within simulations, results revealed that there was little to no oscillatory flow (OSI=0) across the tissue specimens when the scaffold was at constantly straight configurations [6]. Shear stress averages were found to be 1.98 dynes $/ \mathrm{cm}^{2}$ at the inner walls, and $2.43 \mathrm{dynes} / \mathrm{cm}^{2}$ at the outer walls [5].

\subsubsection{Cyclic Flexure and Steady Flow (Flex-Flow)}

Flex-Flow conditions used a steady-state flow regime coupled with cyclic flexing of the samples at the physiologically relevant frequency of $1 \mathrm{~Hz}$. Results demonstrate that 
the OSI=0.11 at the inner walls of the samples and OSI=0.09 at the outer walls averaged out to an OSI=0.10. Shear stress averages were found to be $2.91 \mathrm{dynes} / \mathrm{cm}^{2}$ at the inner walls and 4.73 dynes $/ \mathrm{cm}^{2}$ at the outer walls. Use of Flex-Flow showed improved collagenous formation and optimized expression of genes involved with valvular development such as Klf-2a and Cdz-1 [5].

\subsubsection{Non-Physiological Pulsatile Flow (NPFF)}

A square-wave, non-physiological flow regime was prescribed to the inlet of the bioreactor system. When utilized within simulations, results revealed that there was high oscillatory flow with an OSI $=0.45$ at the inner walls and an OSI $=0.29$ at the outer walls averaged out to OSI $=0.37$. Shear stress averages were shown to be $1.5 \mathrm{dynes} / \mathrm{cm}^{2}$ at the inner walls and 1.2 dynes $/ \mathrm{cm}^{2}$ at the outer walls. When used in experiments, NPFF conditions had similar rates of collagenous development and expression of $\mathrm{Cdz}-1$ compared to the Flex-Flow case, but sustained a statistically significant decrease in the expression of Klf-2a [12].

\section{SPECIFIC AIM 1}

Young patients born with critical congenital valve diseases are faced with a grim prognosis. Prosthetic valves, while a mature technology, do not cater to the small size of these patients, nor can they enable somatic growth. Towards the development of functional tissue engineered heart valves (TEHVs) several protocols have been investigated in an effort to optimize physical, mechanical, and biochemical properties [2, 22, 31]. Regardless of the specific approaches, gene, cellular, and tissue-level responses of the TEHV are intimately tied to the mechanical environment native to the 
cardiovascular system $[2,23,32,12]$. Recently, the directionality of fluid-induced shear stress, i.e., oscillatory shear stresses, have been shown to be essential in heart valve tissue development including cellular proliferation, extracellular matrix (ECM) production, and the overall preservation of the valvular phenotype [33-35]. In an in vivo model, Vermot et al established a link between OSS and its direct regulatory role on the critical gene KLF2A, whose absence results in heart valve defects [7]. In our laboratories, as well as elsewhere, in vitro mechanical environments that elicit OSS, such as Flex-Flow or pulsatile flow, demonstrated clear merits of engineered extracellular matrix (ECM) collagen production and valvular phenotype derived from human bone marrow mesenchymal stem cells (HBMSCs) [14, 5, 12]. In particular when the mechanical conditions were in the physiological range (fluid shear stresses of 4-5 dynes $/ \mathrm{cm}^{2}$ and cyclic flexure frequency of $1 \mathrm{~Hz}$ ), de novo tissue content was further augmented, with relatively-high presence of genes supporting the cardiovascular phenotype, including robust KLF2A expression [5]. On the other hand, use of a NPPF condition adversely impacted KLF2A expression, although other pertinent genes (such as FZD2) and overall ECM collagen content remained unaffected. Regardless of the specific mechanical conditions used, the common underlying thread in studies where tissue construct biological properties were substantially improved in comparison to static culture, was the exposure of BMSC-derived engineered tissues to OSS. Indeed, we previously established that OSS plays a critical role in the formation of engineered heart valve tissues when the magnitude of fluid-induced shear stresses is physiologically relevant [12]. In this investigation, we wanted to identify the specific value(s) of fluid oscillations rather than the shear stresses that led to these enhanced properties. He and 
$\mathrm{Ku}[20]$ have shown that the fluid oscillations can be quantified using the oscillatory shear index (OSI) (Eq. 1).

Utilizing physiologically relevant hemodynamic conditions from different vascular sources, here, we took a computational approach towards determining a range of OSI that may be important in enhancing gene, cell, and tissue-level properties in TEHVs. Subsequently the effect of physiologically relevant OSI versus non-physiological OSI, either too high or too low, was evaluated experimentally in HBMSC culture experiments.

\subsection{Methodology of Specific Aim 1}

All simulations in this study were carried out using commercially available software (ANSYS® Workbench 2016, Ansys Inc., Canonsburg, PA) within a Windows ${ }^{\circledR} 7$ 64-bit Operating System environment (Microsoft Inc., Redmond, WA). A workstation with dual processors was used to facilitate an efficient and accurate solution convergence (Intel Xeon® X5550, 2.66 GHz processor speed, Intel Inc., Santa Clara, CA).

The simulations comprised a geometry depicting a flow-stretch-flexure (FSF) bioreactor which has been used to conduct several tissue engineering experiments in our laboratory $[25,5,6,12]$. The bioreactor chamber consists of a U-shaped flow path, with a diameter of $13 \mathrm{~mm}$. Recognizing the significance of wall shear stress induction regarding heart valve tissue development, there have been several cases of flow rate that have been analyzed in the application to the FSF bioreactor, including static, steadystate, pulsatile square waveform, cyclic flexure, and Flex-Flow cases.

For each physiologically relevant pulsatile flow simulation that was run, a no-slip boundary condition was assigned to the walls of the bioreactor while a porous fluid 
interface was prescribed to the surfaces of each rectangular-shaped scaffold $(n=3$ specimens per flow chamber; specimen dimensions: $17 \mathrm{~mm}$ x $6.5 \mathrm{~mm}$ x $1 \mathrm{~mm}$ ). Three pulsatile flow simulations were conducted; specifically, the inlet pulsatile velocity boundary conditions from two arteries (pulmonary artery (Fig. 2) and aorta (Fig. 3)) [Lotz et al. 2002] and one vein (superior vena cava (Fig. 4)) [36] were used. A zeropressure boundary condition was prescribed at the outlets. All simulations utilized fluid material properties of basal cell culture media consisting of a density of $1.01 \mathrm{~g} / \mathrm{cm}^{3}$ and a dynamic viscosity of $1.27 \mathrm{cP}[6]$. Solution convergence was interpreted to have occurred when residuals arising from the continuity and momentum equations were able to meet a prescribed convergence criterion of $1 \times 10^{-9}$.

\subsubsection{Inlet and Outlet Boundary Conditions}

For the following simulations, three physiological boundary conditions [36] were separately prescribed across the bioreactor inlet while a zero-pressure boundary condition was prescribed to the outlet.

\subsubsection{Pulmonary Arterial Waveform}

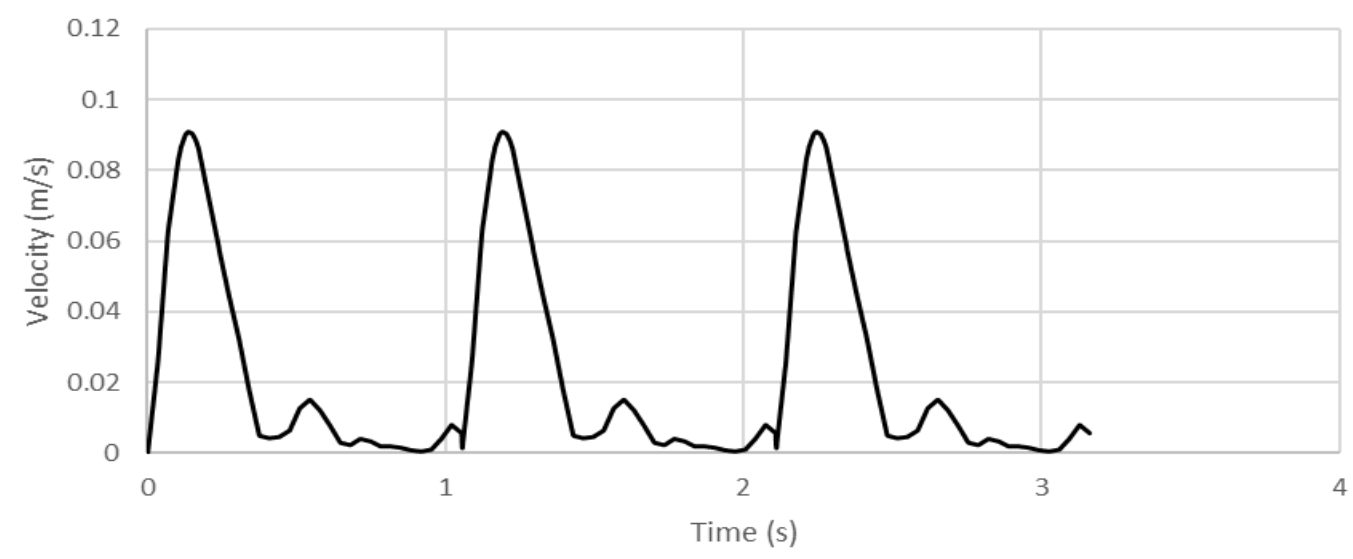

Figure 2: Pulmonary arterial waveform with peak velocity at $0.095 \mathrm{~m} / \mathrm{s}$ and a mean velocity at $0.022 \mathrm{~m} / \mathrm{s}$ 


\subsubsection{Aortic Waveform}

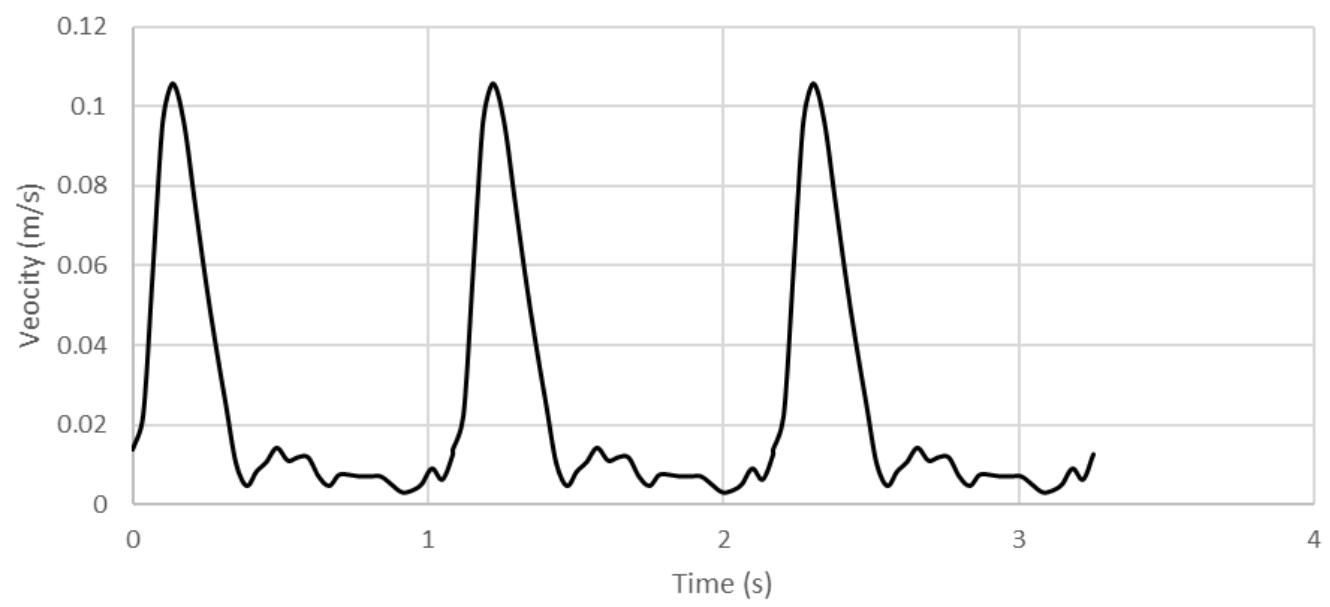

Figure 3: Aortic waveform with peak velocity at $0.109 \mathrm{~m} / \mathrm{s}$ and a mean velocity at $0.0241 \mathrm{~m} / \mathrm{s}$.

\subsubsection{Superior Vena Caval Waveform}

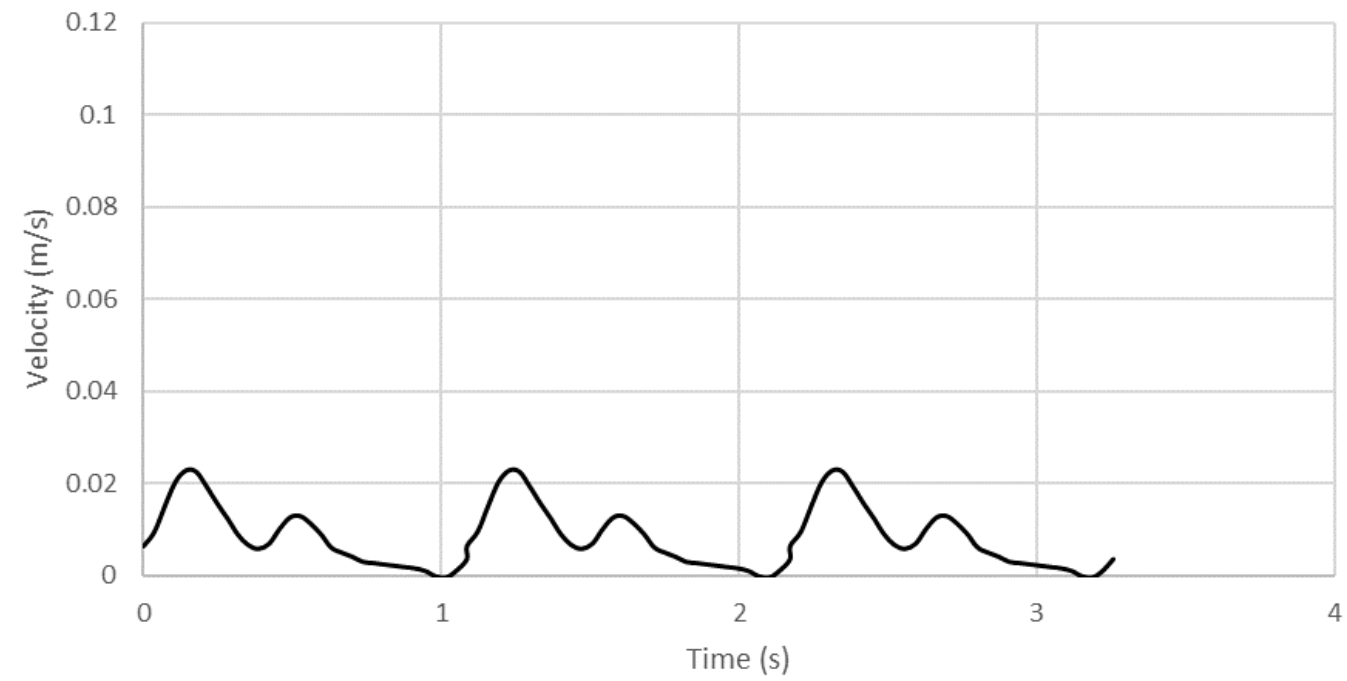

Figure 4: Superior vena caval waveform with peak velocity at $0.025 \mathrm{~m} / \mathrm{s}$ and a mean velocity at 0.0081 $\mathrm{m} / \mathrm{s}$. 


\subsubsection{Numerical Independence}

Independence testing was carried out to optimize the computational conditions as follows:

\subsubsection{Mesh Independence}

A bioreactor geometry mesh that we had previously utilized for computational investigations [6] was used as the basis for the current study (Fig. 5). The optimized quadrilateral meshing density for the geometry consisted of 441,000 elements and 472,000 nodes. Use of this mesh in a steady flow simulation in comparison to our previous work [13] yielded a mean specimen wall shear stress ( $\mathrm{n}=3$ specimens) that was within a 5\% margin of agreement.

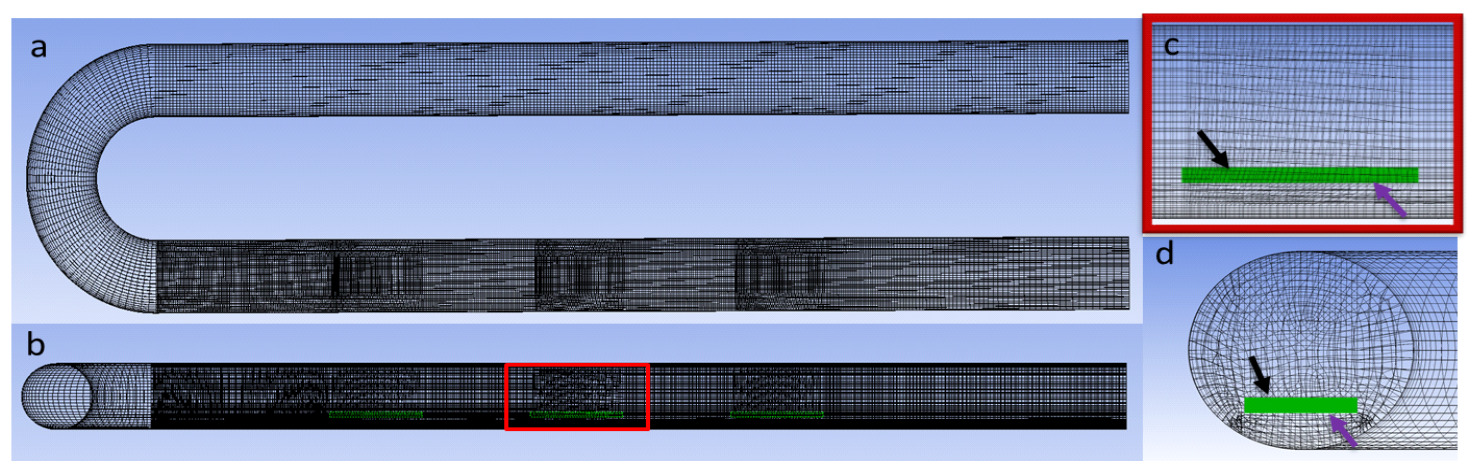

Figure 5: The FSF bioreactor quadrilateral mesh as shown from both the xz-axis (a) and the yz-axis (b). The mesh density of the geometry was optimized at $4.72 \times 105$ nodes and $4.41 \times 105$ elements with the mesh refined around the samples of the bioreactor, as depicted from the xz-axis (c) and the xy-axis (d) with the black arrow pointing to the proximal wall of each respective sample and the purple arrow pointing to the distal wall.

\subsubsection{Timestep Independence}

The pulmonary artery computational fluid dynamics (CFD) simulation was used as the basis to select a valid time-step for all simulation cases. A time-step of $1 \mathrm{~ms}$ yielded a low Courant number $(<1.00)$ suggesting that it would permit temporal accuracy in 
resolving the flow computations. However, to facilitate efficiency in computation times for solution convergence, the time-step was gradually increased ( $2 \mathrm{~ms}$ and $3 \mathrm{~ms})$ to determine if a solution was possible to within 5\% error of the solution derived from the 1-ms time-step case. Eventually, a 2-ms time-step was chosen based on its resulting time-averaged specimen shear stress prediction ( $\mathrm{n}=3$ specimens), which was found to be within $5 \%$ of the value obtained for the $1 \mathrm{~ms}$ time step simulation.

(a)

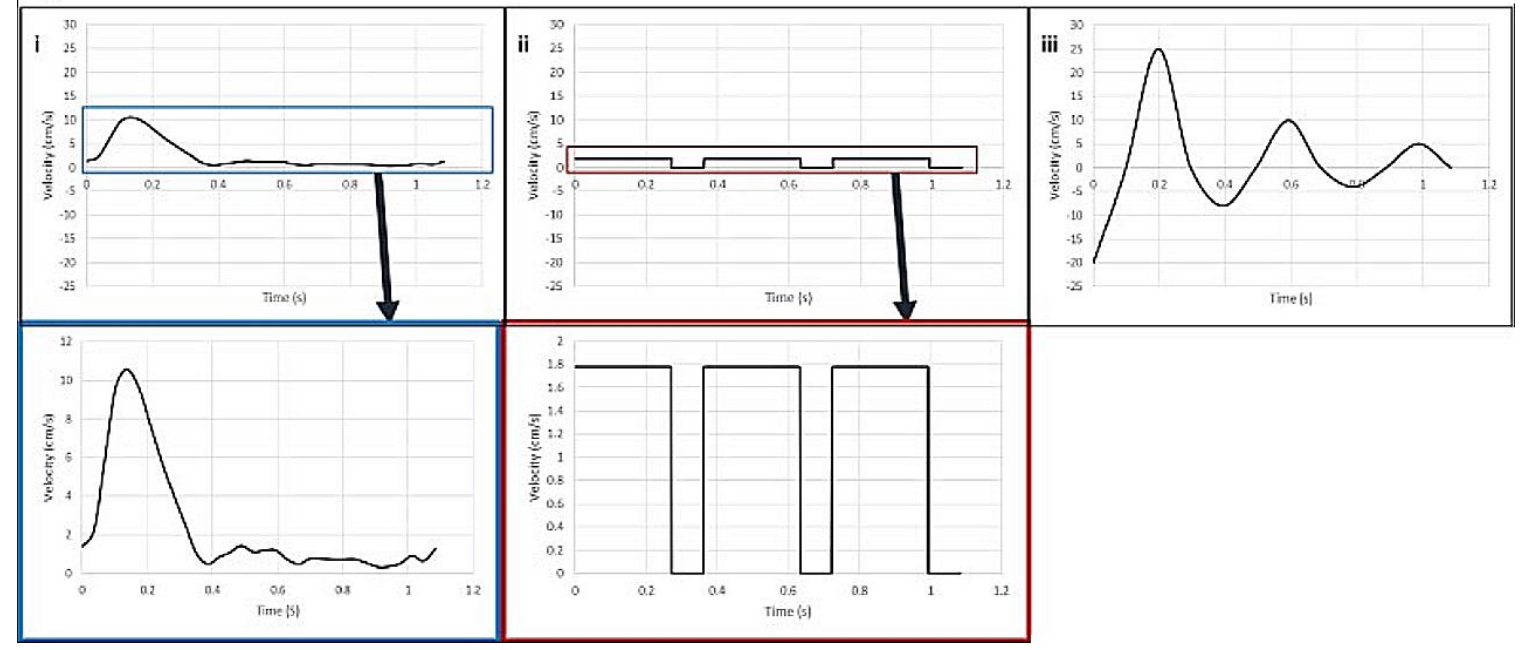

(b)

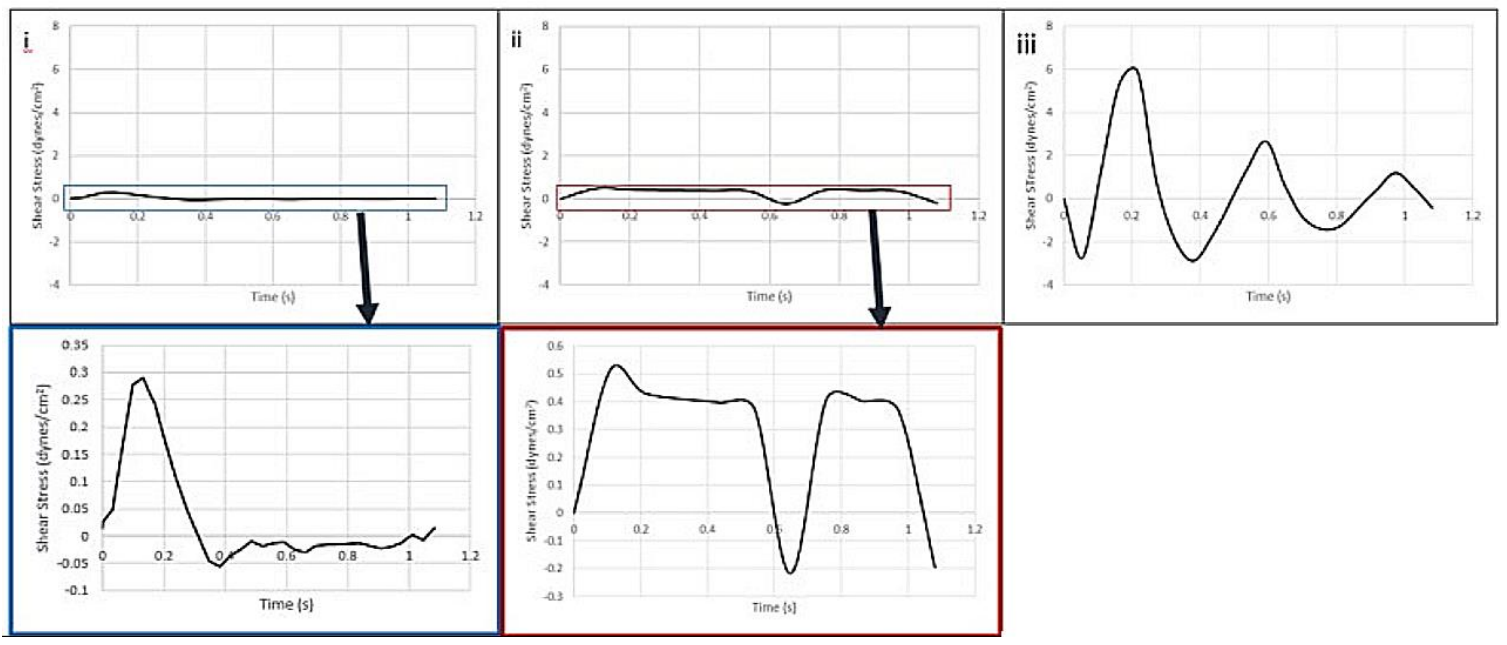


Figure 6: Velocity waveforms (a) that produced corresponding shear stress profiles (b) to obtain a (i) physiologically relevant OSI (OSI $=0.23)$ (ii) low-OSI $(\mathrm{OSI}=0.10)$ and (iii) high-OSI $(\mathrm{OSI}=0.38)$. Both (i) and (ii) show velocity waveforms and shear stress profiles at magnified scales, as indicated by black arrows.

\subsubsection{Cyclic Independence}

In the pulmonary artery CFD simulation the time-averaged specimen wall shear stresses $(n=3)$ of the 1 st and 2 nd cycles were compared to their corresponding value in the 3rd cycle. Both the 1 st and 2 nd cycles yielded values that were within $1 \%$ of the time-averaged specimen shear stress value obtained from the 3rd cycle. Therefore, all results presented in this study were taken from the 2 nd cycle.

\subsubsection{CFD Validation}

Results in the current study from the pulmonary artery pulsatile flow simulation utilizing a structured, quadrilateral mesh were compared to a previously well-established pulsatile flow simulation from our laboratory which used a tetrahedral, unstructured mesh [13]. Again, the computed time-averaged specimen wall shear stress metric was used as the basis for the comparison. The time-averaged specimen shear stress from the structured mesh was found to be within 5\% of the value from the unstructured mesh.

\subsubsection{Mechanobiological Testing}

First, additional FSF bioreactor simulations were performed using a set of prescribed velocity waveforms (Fig. 6a) which produced time-averaged shear stress profiles (Fig. 6b) that were subsequently used to compute three OSI-values representing low $(\mathrm{OSI}=0.10)$, high $(\mathrm{OSI}=0.38)$ and the sweet-spot $(\mathrm{OSI}=0.23)$ ranges. The physiologically relevant and high OSI were obtained from the distal wall of the specimen and low OSI from the proximal wall. 
Next, in cell culture experiments, $~ 1$ million HBMSCs (Fisher Scientific, Pittsburgh, PA) were cultured up to passage 4 (P4). Cells between P4-P6 were subsequently utilized and plated in bovine-derived fibronectin (Sigma Aldrich St. Louis, MO) coated microfluidic channels and were subjected to three different OSI groups using a commercially-available shear stress cell assay system available in our laboratory (Fluxion Biosciences, South San Francisco, CA; Martinez et al. 2012). HBMSCs were subjected to a 48-hour conditioning cycle and were subsequently processed for RNA isolation. A fourth group, a "No flow" or HBMSCs grown in static culture conditions, was used as a control. Each group comprised of 4 samples, where each sample was pooled from 2 microfluidic channels.

\begin{tabular}{lll}
\hline Gene list & Forward primer & Reverse primer \\
\hline GAPDH & AGCCACATCGCTCAGACAC & GCCCAATACGACCAAATCC \\
KIf2a & CCGTCTGCTTTCGGTAGTG & AAGAGTTCGCATCTGAAGGC \\
CD31 & CCAAGGTGGGATCGTGAGG & TCGGAAGGATAAAACGCGGTC \\
a-SMA & TCAATGTCCCAGCCATGTAT & CAGCACGATGCCAGTTGT \\
TGFb1 & GCGTGCTAATGGTGGAAAC & CGGTGACATCAAAAGATAACCAC \\
NOTCH1 & GTGACTGCTCCCTCAACTTCAAT & CTGTCACAGTGGCCGTCACT \\
VCAM1 & GCTCTGTGACCATGACCTGTTC & CTGACCAAGACWTTGTATCTC \\
BMP2 & CGGTCTCCTAAAGGTCGACCAT & CGAACTTCCTGCGGCCCAGCT \\
Osteocalcin & CACTCCTCGCCCTATTGGC & CCCTCCTGCTTGGACACAAAG \\
FGF4 & CCAACAACTACAACGCCTACGA & CCCTTCTTGGTCTTCCCATTCT
\end{tabular}

Table 1: Quantitative real-time polymerase chain reaction primer sequences for gene expression analysis of HBMSCs.

\subsubsection{Gene Expression Analysis}

After 48-hours of conditioning, HBMSCs were subjected to gene expression assessment. In brief, total RNA was isolated according to manufacturer's protocol (RNeasy Micro kit, Qiagen) and was quantified using NanoDrop 2000c spectrophotometer (Thermo Fisher Scientific, Pittsburgh, PA). 200ng of total RNA was 
used for the cDNA synthesis RevertAid First Strand cDNA Synthesis kit (Thermo

Fisher, Waltham, CA). The primers (Table 1) were designed using Primer3 software and gene sequences were obtained from Basic Local Alignment Search Tool (BLAST) program, National Center for Biotechnology Information (NCBI). Quantitative real-time polymerase chain reaction (qRT-PCR) was performed using a commercially available kit Maxima SYBER Green/ROX qPCR Master Mix (Thermo Fisher Scientific, Pittsburgh, PA). Signals were detected with the Step-One Real-Time PCR System (Applied Biosystems). The change in cycle threshold $(\Delta \mathrm{Ct})$ values were averaged and normalized with GAPDH, an endogenous gene using the $\Delta \Delta \mathrm{Ct}$ method [Schmittgen and Livak 2008]. Untreated HBMSCs were used as a control for data analysis.

\subsubsection{Statistical Analysis}

Shear stress and OSI findings on housed-bioreactor specimens $(n=3)$ were presented as the mean \pm standard error of the mean (SEM). An ANOVA analysis was performed for the 5 groups compared, namely, the three physiologically relevant pulsatile flow cases conducted here, and from the previously performed Flex-Flow [5] and NPPF [12]studies. A Tukey's post hoc test was conducted followed by a one-way ANOVA to assess any significance between the groups (SPSS, V16, IBM, Armonk, NY). A statistically significant difference between groups was determined to have occurred when $\mathrm{p}<0.05$.

\subsection{Results of Specific Aim 1}

\subsubsection{Velocity Streamlines}

Velocity contours were taken from cross-sectional planes of the bioreactor across the proximal and distal walls of the scaffolds (fig. 7-9). In verifying of proper coupling of 
the profile to the inlet of the bioreactor, the contours of the planes for each time point follow in accordance with the acceleratory and deceleratory phases of the waveform for each profile case. The bottom plane, where the distal walls are taken into account, has a notably higher velocity distribution than the top plane of each profile case.

\subsubsection{Pulmonary Artery}

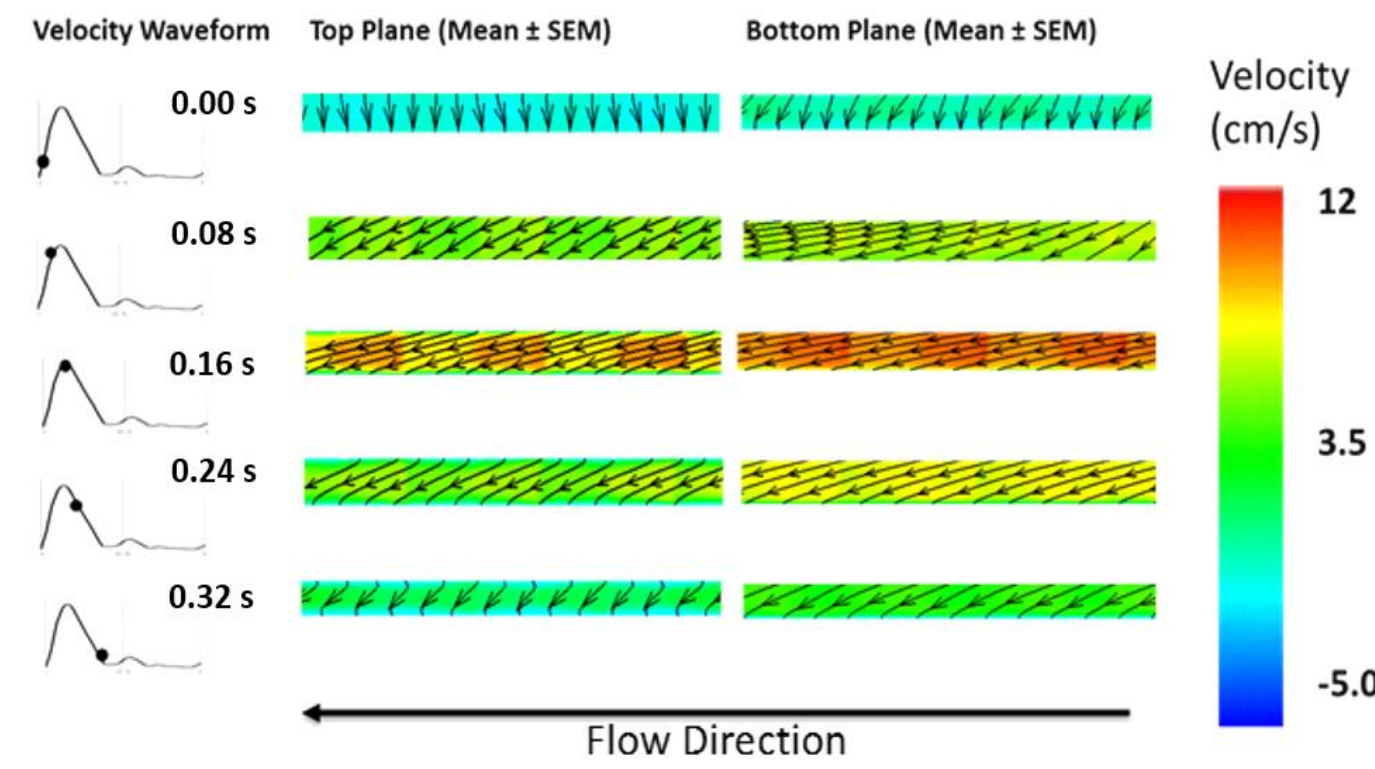

Figure 7: Instantaneous velocity contours of the pulmonary arterial profile under five equitemporal time points from both the top plane (proximal walls) and the bottom plane (distal walls). Peak velocity is distinguished at the peak of the waveform at $0.16 \mathrm{~s}$. 
3.2.1.2. Aorta

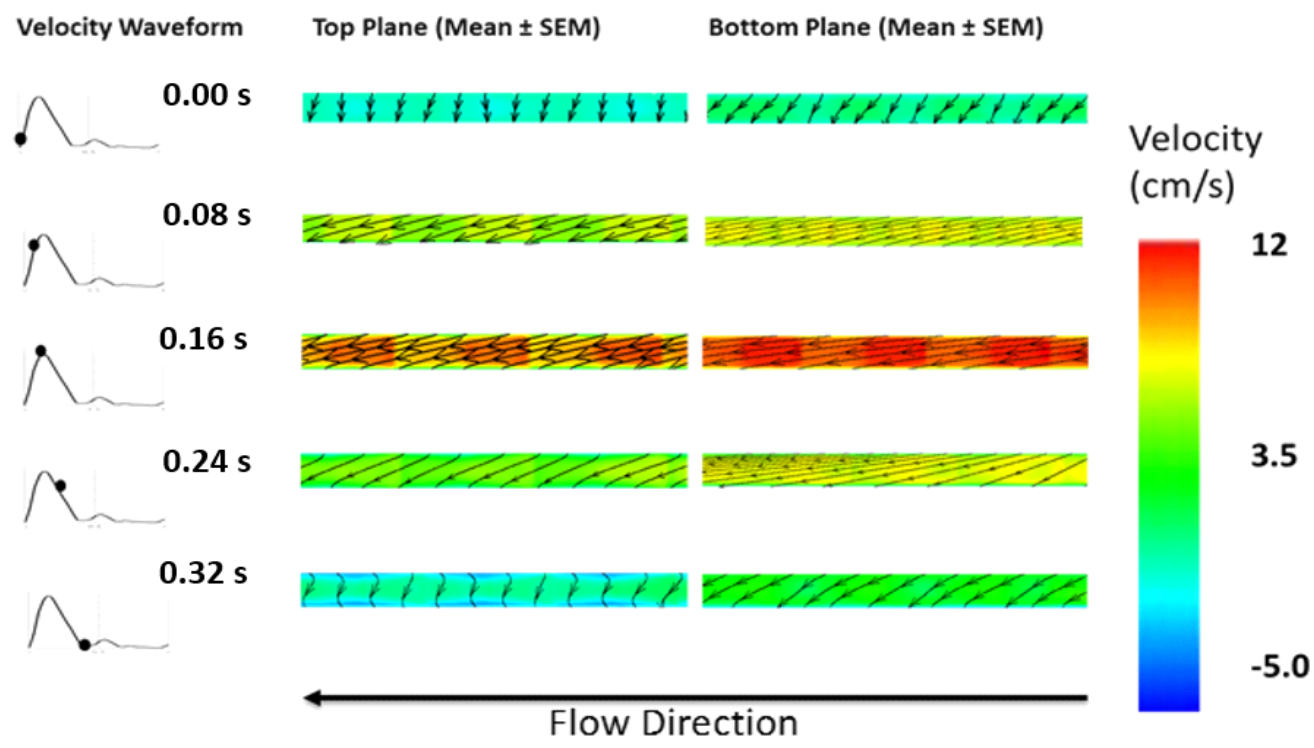

Figure 8: Instantaneous velocity contours of the aortic profile under five equitemporal time points from both the top plane (proximal walls) and the bottom plane (distal walls). Peak velocity is distinguished at the peak of the waveform at $0.16 \mathrm{~s}$. 


\subsubsection{Superior Vena Cava}

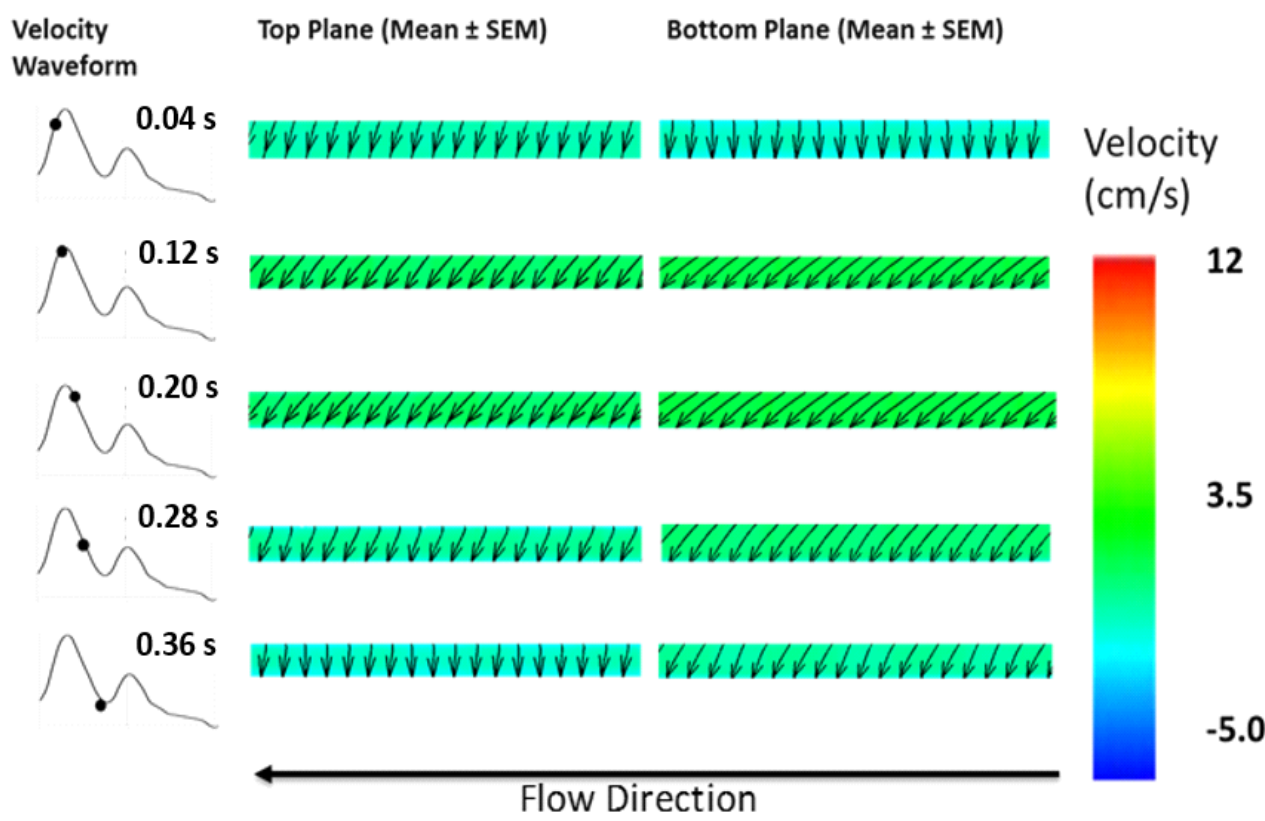

Figure 9: Instantaneous velocity contours of the superior vena caval profile under five equitemporal time points from both the top plane (proximal walls) and the bottom plane (distal walls). Peak velocity is distinguished at the peak of the waveform at $0.12 \mathrm{~s}$.

\subsection{2. $\underline{\text { Vorticity Plots }}$}

Vortices and eddy currents were quantified on cross-sectional contour planes intersecting across the middle of the second scaffold within the bioreactor (Fig. 10-12). Trends in high magnitude vortices were observed notably around the scaffold juncture of the contour maps for each profile case analyzed with peak magnitude vorticity densities occurring during the deceleratory phase of each profile case, when flow reversal would start to manifest. 


\subsubsection{Pulmonary Artery}

\begin{tabular}{|l|l|}
\hline Waveform Position & Contour \\
\hline $0.00 \mathrm{~s}$ & $\mathrm{~s}$ \\
\hline $0.04 \mathrm{~s}$ & $\mathrm{~s}$ \\
\hline $0.28 \mathrm{~s}$ & \\
\hline
\end{tabular}

\section{Vorticity $(\mathrm{Hz})$}
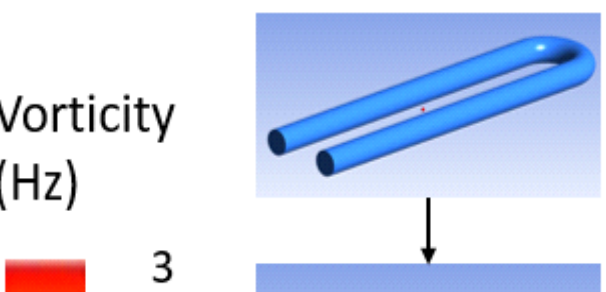

3
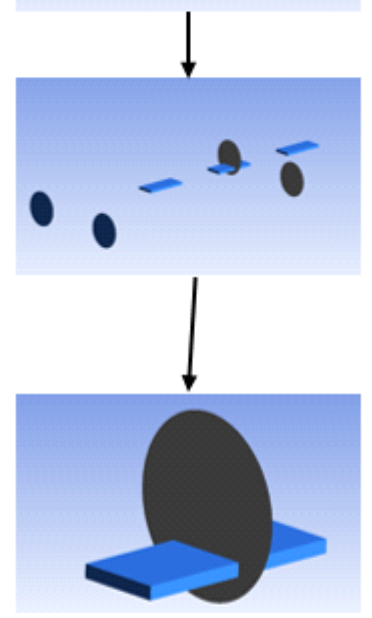

0

Figure 10: Sample-intersecting vorticity contour maps of the pulmonary arterial profile at seven time points. 


\subsubsection{Aorta}

\begin{tabular}{|c|c|}
\hline Waveform Position & Contour \\
\hline $0.00 \mathrm{~s}$ & \\
\hline $0.04 \mathrm{~s}$ & \\
\hline $0.08 \mathrm{~s}$ & \\
\hline $0.16 \mathrm{~s}$ & \\
\hline $0.24 \mathrm{~s}$ & \\
\hline $0.32 \mathrm{~s}$ & \\
\hline $1.36 \mathrm{~s}$ & \\
\hline
\end{tabular}

\section{Vorticity}

(Hz)

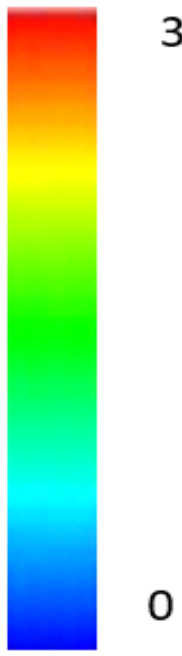

Figure 11: Sample-intersecting vorticity contour maps of the aortic profile at seven time points. 


\subsubsection{Superior Vena Cava}

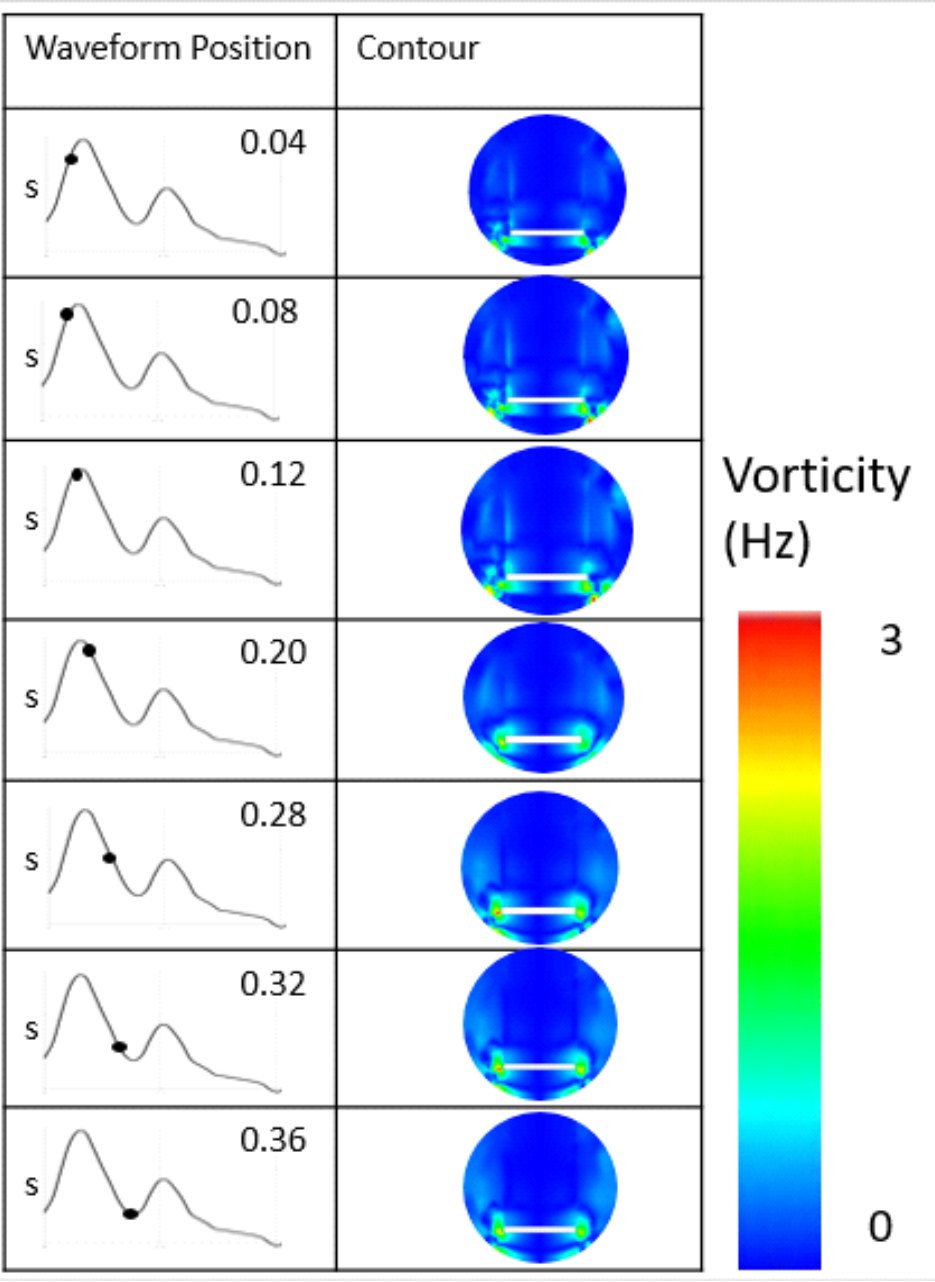

Figure 12: Sample-intersecting vorticity contour maps of the superior vena caval profile at seven time points.

\subsubsection{Instantaneous Axial Wall Shear Stress Approximations}

The spatial distributions of shear stresses were presented for both the distal and proximal walls of the bioreactor specimens at the specific time points of the cardiac cycle (Fig. 13-15). The cardiac cycle durations for the pulmonary artery, aortic, and superior vena cava were $1.08 \mathrm{~s}, 1.05 \mathrm{~s}$ and $1.08 \mathrm{~s}$ respectively [Lotz et al. 2002]. In all 
simulations carried out, maximum specimen shear stresses corresponded with the peak temporal position of the prescribed pulsatile velocity profile as expected. Also in general, shear stresses were higher on the distal side of the specimens as compared to the proximal side.

\subsubsection{Pulmonary Artery}

In the pulmonary artery case, during the early acceleration phase $(t=1.08 \mathrm{~s})$ of the cardiac cycle, the resulting shear stresses were not uniformly distributed (Fig. 13). However sufficient inertial forces at a time instance $(\mathrm{t}=1.12 \mathrm{~s})$ proximal to the peak velocity temporal position enabled higher shear, streamlined laminar flow. During the latter stages of flow deceleration, reversal of flow was observed (at $t=1.36 \mathrm{~s}$ ). 


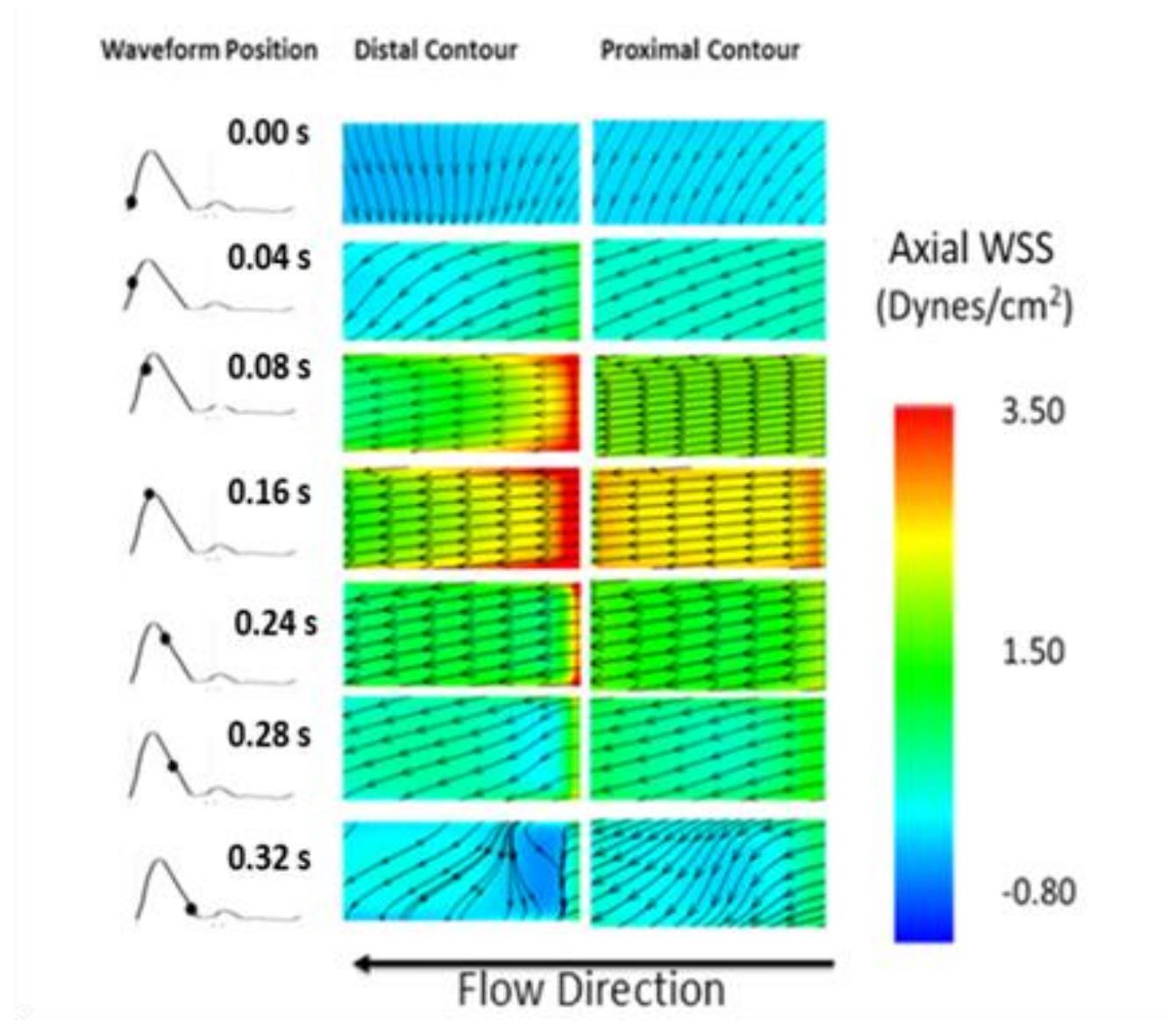

Figure 13: Instantaneous sample shear stress contours for pulmonary arterial simulation. For time points $0.00 \mathrm{~s}, 0.04 \mathrm{~s}, 0.08 \mathrm{~s}, 0.16 \mathrm{~s}, 0.24 \mathrm{~s}, 0.28 \mathrm{~s}$ and $0.32 \mathrm{~s}$, the shear stress averages were $0.08 \pm$ $0.002,0.46 \pm 0.009,1.8 \pm 0.02,2.5 \pm 0.02,1.3 \pm 0.01,0.76 \pm 0.009$ and $0.28 \pm 0.007$, respectively.

\subsubsection{Aorta}

The aortic velocity waveform-derived specimen shear stress distributions evolved similarly to the pulmonary artery case (Fig. 14). However, the aortic shear stresses increased in magnitude more rapidly, reaching a maximum value at $\mathrm{t}=1.20 \mathrm{~s}$ as opposed to the pulmonary artery simulation $(\mathrm{t}=1.24 \mathrm{~s})$; similarly, noticeable flow deceleration effects on specimen shear stress were occurred sooner in the aorta (at $t=1.32 \mathrm{~s}$ comparing Fig 3a to 3b). 


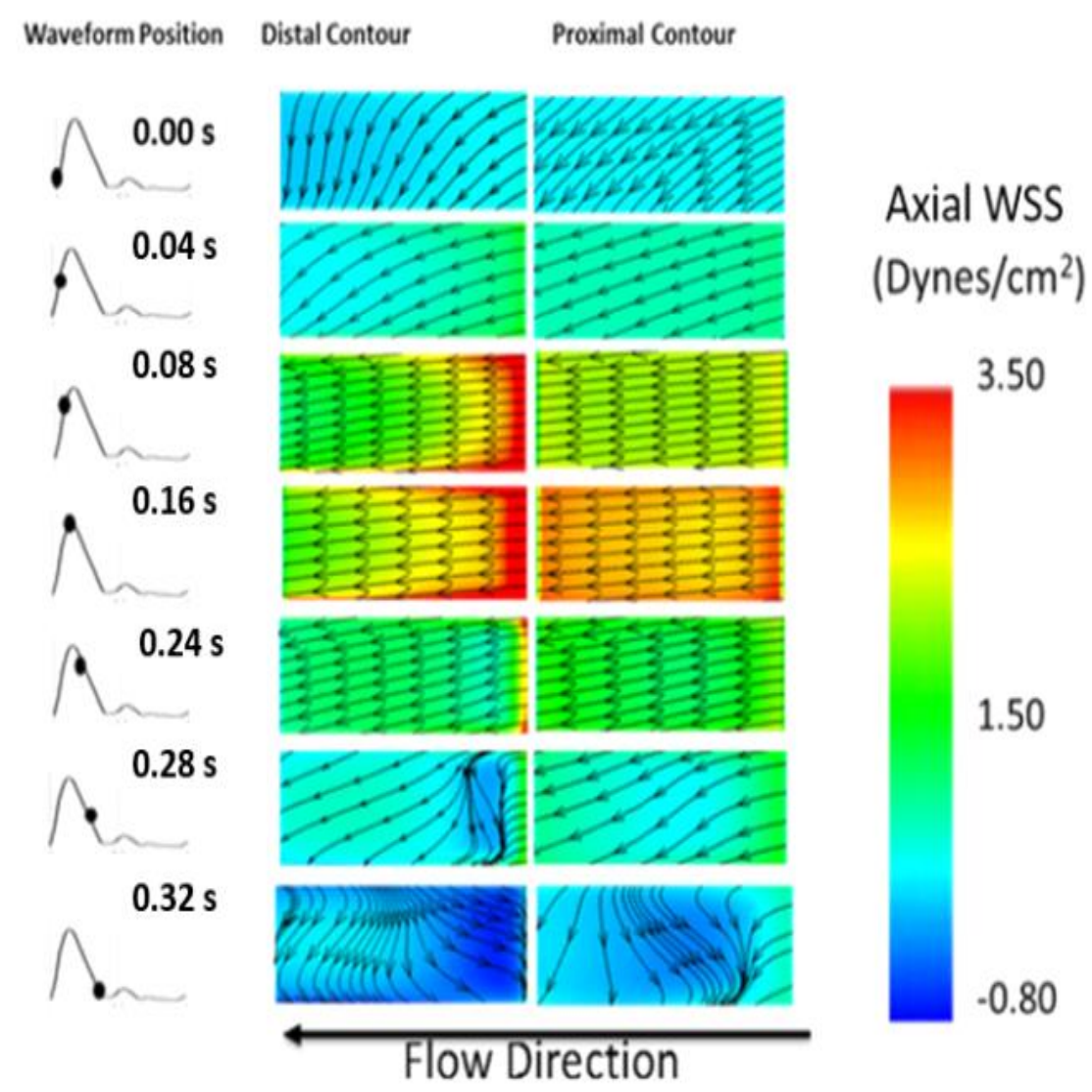

Figure 14: Instantaneous sample shear stress contours for aortic simulation. For time points 0.00 s, $0.04 \mathrm{~s}, 0.08 \mathrm{~s}, 0.16 \mathrm{~s}, 0.24 \mathrm{~s}, 0.28 \mathrm{~s}$ and $0.32 \mathrm{~s}$, the shear stress averages were $0.21 \pm 0.003,0.59$ $\pm 0.006,2.0 \pm 0.02,2.7 \pm 0.02,1.2 \pm 0.01,0.49 \pm 0.009$, and $-0.06 \pm 0.008$, respectively.

\subsubsection{Superior Vena Cava}

As would be expected, the bulk specimen shear stress ranges were considerably lower in venous flow conditions (Fig. 15) as opposed to arteries (Fig. 13 and 14). Multiple temporal positions at and beyond the mid-point of the deceleration phase $(t=1.36 \mathrm{~s})$ revealed substantial flow disturbances as well as flow reversal from the principal flow direction. 


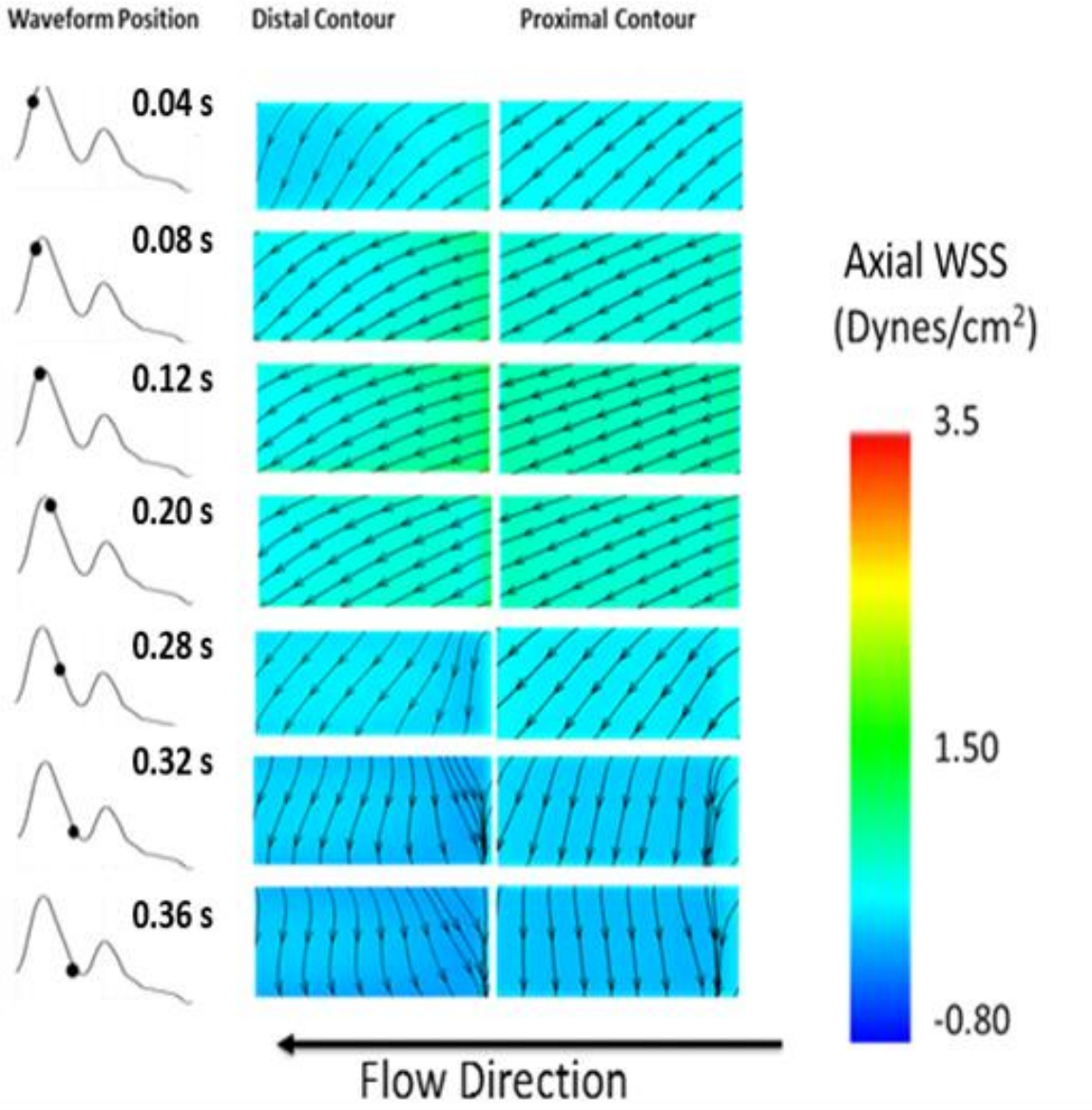

Figure 15: Instantaneous sample shear stress contours for superior vena cava simulation. For time points $0.04 \mathrm{~s}, 0.08 \mathrm{~s}, 0.12 \mathrm{~s}, 0.20 \mathrm{~s}, 0.28 \mathrm{~s}, 0.32 \mathrm{~s}$, and $0.36 \mathrm{~s}$, the shear stress averages were 0.024 $\pm 0.0003,0.043 \pm 0.0004,0.057 \pm 0.0004,0.047 \pm 0.0003,0.017 \pm 0.0002,0.004 \pm 0.0002$, and $0.003 \pm 0.0002$ respectively.

\subsubsection{Oscillatory Shear Stresses}

OSI distributions for both the proximal and distal walls were plotted (Fig. 16) for all three CFD simulation cases of physiologically relevant pulsatile flow and compared to our previous findings utilizing a non-physiological square waveform [12] and a combination of physiological-scales of cyclic flexure $(1 \mathrm{~Hz})$ and steady flow (FlexFlow; flow rate of $850 \mathrm{ml} / \mathrm{min}$ ) [5, 6]. The overall OSI (Mean $\pm \mathrm{SEM}$ ) for the entire 
specimen ( $\mathrm{n}=3$; i.e., considering both proximal and distal sides) for the pulmonary arterial, aortic, superior vena cava, square pulsatile, and Flex-Flow conditions were as follows: $0.23 \pm 0.002,0.18 \pm 0.002,0.19 \pm 0.001,0.37 \pm 0.021$, and $0.1055 \pm 0.052$, respectively. Therefore, the range of OSI was found to be: $0.18 \leq \mathrm{OSI} \leq 0.23$ across the three physiological pulsatile flow waveforms investigated. This range was found to be significantly lower and higher to non-physiological and non-pulsatile flow-induced OSI (Flex-Flow) cases respectively $(\mathrm{p}<0.05)$.

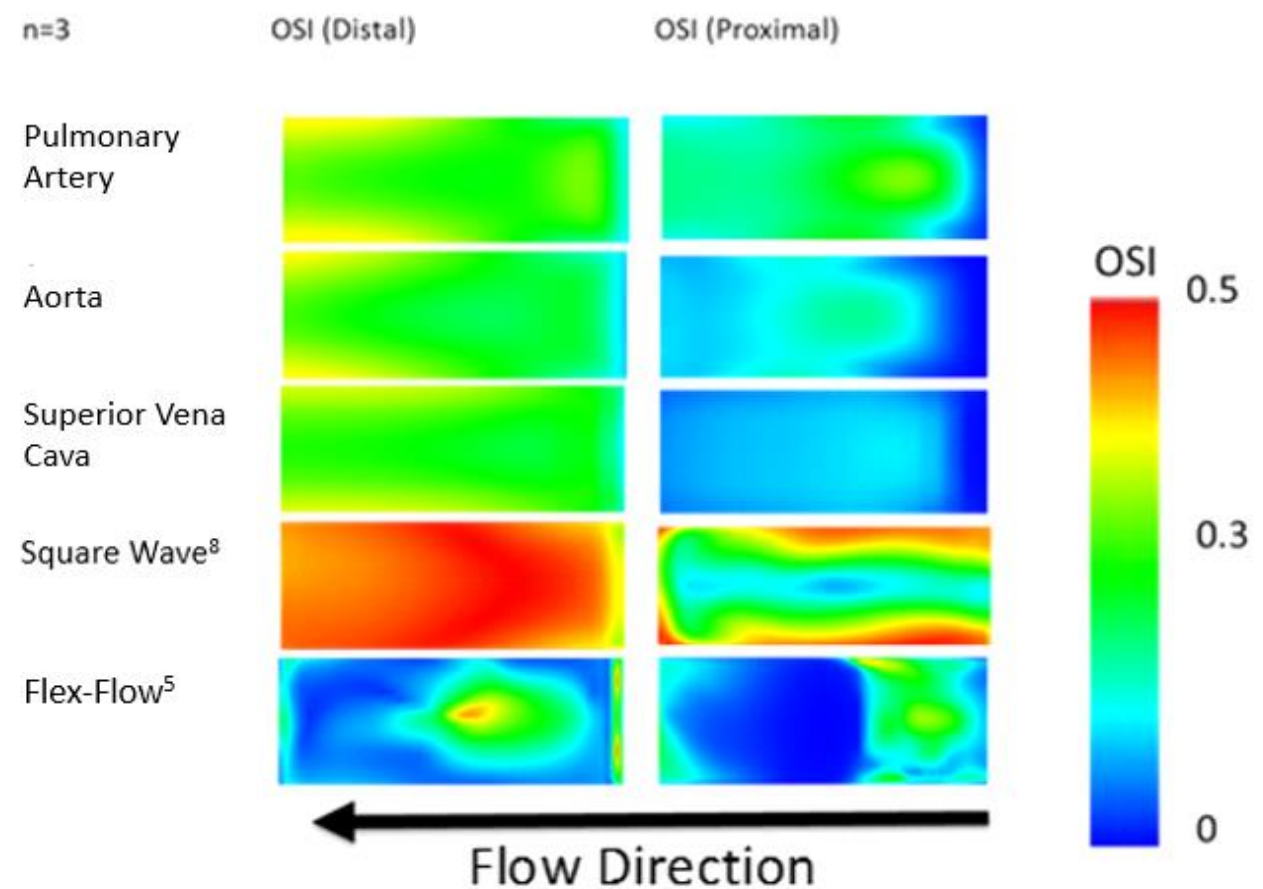

Figure 16: OSI contours $(\mathrm{n}=3)$ of the samples housed within the FSF bioreactor. The mean OSI of the distal walls for the pulmonary arterial, aortic, superior vena cava, square wave, and Flex-Flow conditions were $0.28 \pm 0.002,0.25 \pm 0.002,0.27 \pm 0.001,0.45 \pm 0.023$, and $0.117 \pm 0.003$, respectively. On the other hand, the corresponding mean OSIs for the proximal wall were as follows: $0.18 \pm 0.002,0.10 \pm 0.002,0.11 \pm 0.001,0.29 \pm 0.019$, and $0.094 \pm 0.10$, respectively. 


\subsubsection{Analysis of Gene Expression}

A significantly higher expression $(\mathrm{p}<0.05)$ of valve developmental genes KLF2A and BMP2, as well as significantly greater up-regulation $(\mathrm{p}<0.05)$ of endothelial and activated interstitial markers CD31 and $\alpha$-SMA respectively, were found in the physiologically relevant "sweet-spot" OSI samples in comparison to the low and high OSI groups (Fig. 17a). On the other hand, the expression of the bone or calcification marker, osteocalcin was statistically insignificant $(\mathrm{p}>0.05)$ between the three groups (Fig 17b).

NOTCH1, a valve development gene, was significantly down-regulated $(\mathrm{p}<0.05)$ in the low OSI group as compared to the high OSI and physiologically relevant "sweetspot” OSI groups, which were both modestly up-regulated (Fig 17c). In addition, there was significant down-regulation of TGFb1 (associated with valve calcification) in the low and "sweet-spot" OSI groups $(\mathrm{p}<0.05)$ in comparison to their high OSI counterpart, which was upregulated. Finally, no statistically significant differences ( $\mathrm{p}>$ 0.05) were observed in the VCAM1 expression, a known inflammatory marker (Fig. 17a). 

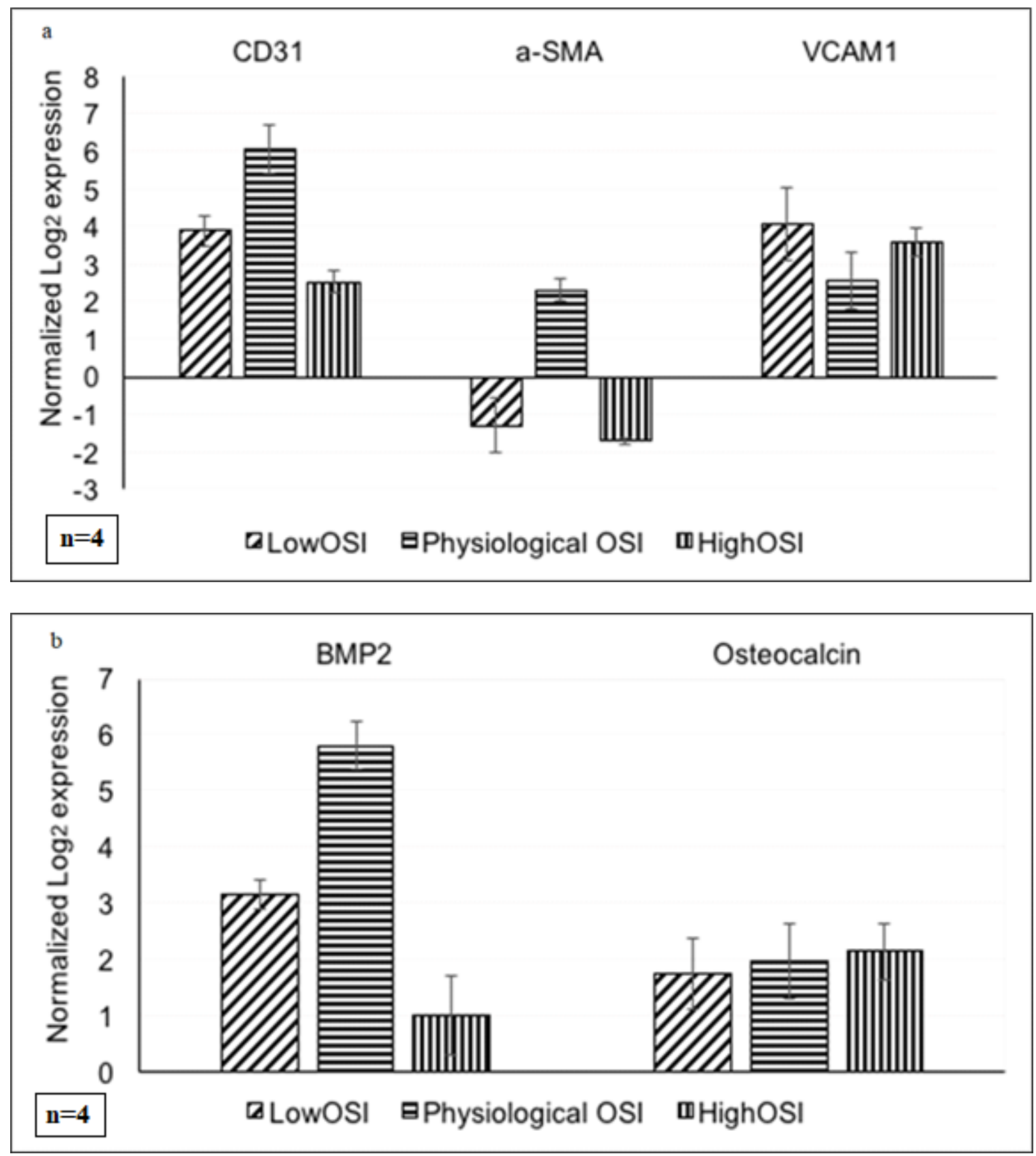


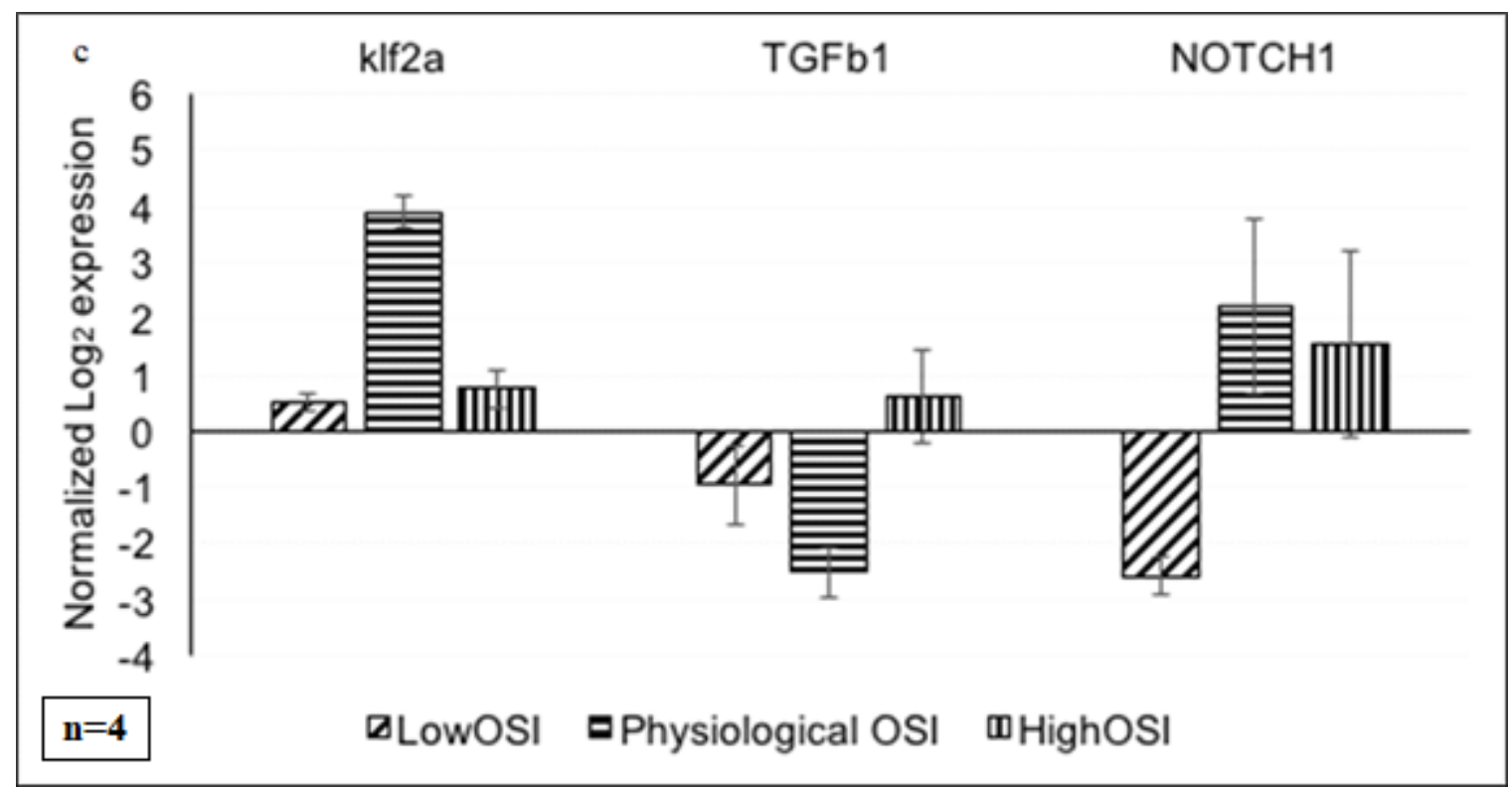

Figure 17: Gene expression following 48 hours of HBMSCs exposed to low OSI (OSI $=0.10)$, high OSI (OSI = 0.38), and physiologically relevant "sweet-spot" OSI (OSI =0.23). Error bars are displayed as \pm SEM; ( $n=4$ samples/group). (a) A statistically significant ( $p<0.05$ ) expression of CD31, $\alpha$-SMA was found in the physiologically relevant OSI-treated group as compared to the low and high OSI counterparts. (b) A significantly higher expression ( $\mathrm{p}<0.05)$ of BMP2 was also observed in the physiologically relevant OSI groups as compared to the other two groups. No significant differences ( $p$ $>0.05$ ) were found to occur in the expression of osteocalcin between the groups. (c) KLF2A and NOTCH1 genes exhibited a significantly higher expression $(p<0.05)$ in the physiologically relevant OSI group. Finally, TGFb1 was significantly down-regulated in the low OSI and physiologically relevant groups $(\mathrm{p}<0.05)$ in comparison to high OSI-treatment wherein it was upregulated.

\subsection{Discussion of Specific Aim 1}

Bioreactor-based mechanical conditioning experiments have shown that Flex-Flow conditions and square pulsatile conditions lead to enhanced matrix development and valve phenotype compared to either steady state flow or cyclic flexure alone $[14,5,12]$. Coupling of cyclic flexure and steady flow conditions augments regional specimen oscillatory shear stresses. Studies have demonstrated the critical link between oscillatory shear stress and valve development, whose absence leads to down-regulation of key valvular genes, such as KLF2A, thereby resulting in heart valve defects [7]. The 
mechanisms of gene regulation are not fully delineated; however, it has already been shown that oscillatory flow-induced stimulus activates mechanosensitive ion channels which lead to gene expression critical in valvulogenesis [37]. This indicates that the localized condition surrounding the valve that necessitates development requires an oscillatory flow component. Specifically, OSS-mediated cell stimulation of KLF2A occurs via the trpv4 and trpp 2 calcium channels [37]. We have since shown that the OSI can be used as a single metric that co-relates with benefits in specimen properties observed from stem cell-derived heart valve tissue engineering experiments [6]; Moreover, oscillatory shear stress environments can be mediated through pulsatile flowalone without the need for flexing specimens [13]. In the present investigation, we hypothesized that healthy valve tissues are exposed to a distinct range of OSI, which may be important in maintaining homeostasis, i.e., an OSI "sweet-spot".

Work we conducted previously using square pulsatile conditions [12] within the same flow-stretch-flexure bioreactor exhibited an OSI of $0.29 \pm 0.019$ on the proximal wall and an OSI of $0.45 \pm 0.023$ on the distal wall surface (Fig. 16), with an overall specimen OSI average of $0.37 \pm 0.021$. This indicated significantly higher $(\mathrm{p}<0.05)$ oscillatory shear stresses compared to OSI derived from physiological flow profiles, which was in the order of 0.18 to 0.23 . In addition, this range of OSI was determined across 3 distinct blood vessel flow profiles (pulmonary artery, aorta and superior vena cava).

The context of this narrow OSI range $(0.18 \leq \mathrm{OSI} \leq 0.23)$ is important given that higher oscillatory shear stresses fail to promote upregulation of key valvular genes (again, such as KLF2A), although this does not appear to alter collagen concentration in in-vitro grown engineered tissues [5]. We speculate that phenotype is highly regulated 
by the specific oscillatory shear stress environment (and likely ideal when within the OSI "sweet-spot" range), whereas the ECM content may not as drastically effected by OSI magnitude. To obtain direct evidence of mechanical conditioning effects on HBMSCs using a "sweet-spot" value of OSI, we were subsequently able to demonstrate experimentally that an OSI $=0.23$ led to maximum expression (relative to lower $(\mathrm{OSI}=$ 0.10) and higher OSI (OSI = 0.38) values; Fig. 17) of the valve developmental genes BMP2 and KLF2A. The context of this narrow OSI is important given that higher oscillatory shear stresses failed to promote up-regulation of key valvular genes. KLF2a, an athero-protective transcription factor, is released by endothelial cells, thereby preventing abnormal ECM due to inflammation during valve morphogenesis [37, 7]. BMP2 is involved during the very early stages of myocardial development and is involved in several vital functions during endocardial cushion formation, including ECM synthesis and tissue growth as well as initial mesenchymal cell proliferation and differentiation to promote the valvular phenotype [38-40]. Moreover, exposure of HBMSCs to the sweet-spot OSI range $(\mathrm{OSI}=0.23)$ led to significant $(\mathrm{p}<0.05)$ upregulation of phenotypic markers CD31 (endothelial) and $\alpha$-SMA (interstitial) indicative of enhanced HBMSC differential capacity in vitro as compared to corresponding exposure to relatively lower $(\mathrm{OSI}=0.10)$ and higher $(\mathrm{OSI}=0.38)$ OSI values.

Collectively, the primary biological significance of the "sweet-spot" OSI identified here is to enhance in vitro gene expression and phenotype in HBMSC-based heart valve tissue engineering using a clearly quantified narrow range of OSI values as opposed to utilizing a random selection of oscillatory flow magnitudes. 
Conversely, Flex-Flow conditions (1 Hz cyclic flexure combined with steady flow spatial mean specimen shear stress of 5 dynes $/ \mathrm{cm}^{2}$ ) previously resulted in a distal OSI of $0.117 \pm 0.003$, a proximal OSI of $0.094 \pm 0.10$ and an overall specimen OSI of 0.106 \pm 0.052 [5]. While both the cyclic flexure and shear stress magnitudes were physiologically relevant, the net OSI was not since it was significantly smaller $(\mathrm{p}<0.05)$ than the OSI "sweet-spot" determined here. The biological implications of exposing engineered valve tissues to physiologically relevant scales of OSI is not entirely clear; however, incorporation of oscillatory shear stresses has augmented phenotype and ECM content under previous Flex-Flow conditions [5]. Here, we have found via performing OSI-based HBMSC experiments, that this can be further improved if the OSI can be moved to within the physiological range, i.e., the "sweet-spot" that was identified here. The bioreactor system which we utilized is able to mimic regionally varying shear stresses on the specimen surfaces and is analogous to native tri-leaflet heart valve shear stress distributions [25]. Indeed, the range of OSI $(0.18-0.23)$ identified here is closeto the typical OSI experienced by aortic valve tissues (OSI 0.16) [41]. Previous studies on Flex-Flow conditions by [5] and [6] found that the 3-dimensional coupling of cyclic flexure, cyclic stretch and fluid-induced shear stresses manifest as oscillatory patterns surrounding the localized flow field of the heart valve leaflets. The flow patterns act on the leaflet surface as oscillatory shear stress. Extensive evidence of oscillatory regulation of valvular gene regulation [7], particularly via Trpv4 and Trpp2 ionic channels [37], has previously been reported. Thus, from a biomimetic standpoint, we interpret that the similarity in values we identified in the current study to the OSI of 
native, healthy human aortic valves which maintains valve homeostasis, will enhance engineered valve tissue development and phenotype.

This study has several limitations and assumptions. We only simulated the biofluid dynamics within 3 vascular locations. Moreover, we did not consider the movement of the heart valve leaflets which is likely to alter specific OSI magnitudes. There are some limitations associated with our bioreactor-derived OSI values from CFD; firstly, the FSF bioreactor that we used for the CFD portion of the current study has been shown to recapitulate the flow patterns experienced by the native tri-leaflet aortic valves[42, 25]. Moreover, the averaged OSI across the entire aortic valve surface was previously reported to be an OSI $\sim 0.16$ [41] which is close to the "sweet spot" OSI range (0.180.23) of the current study. However, we note that fluid-induced oscillations are considerably greater on the fibrosa surface as compared to the ventricularis surface and as a result, regional OSI can vary. The "sweet-spot" OSI range reported here and derived from FSF bioreactor CFD simulations, as well as the native aortic valve, represents averaged values for all surfaces and some differences between bioreactor versus native valve-OSI are bound to occur $(\sim 25 \%)$ and thus represents another limitation of this study. Finally, we speculate that the biological responses of cells other than HBMSCs to the OSI "sweet-spot" or physiologically relevant window of OSI ( 0.18 $\leq \mathrm{OSI} \leq 0.23$ ) may not be the same. Moreover, experiments reported herein were performed in monolayer culture and as a result, the extent to which cell-ECM, i.e. 3dimensional, interactions would be regulated by the "sweet-spot" OSI identified here is not known. Nonetheless the current investigation does serve to demonstrate the relative distinctions between physiologically-induced OSI versus non-physiological counterparts 
and established that the physiological range needed to promote valvular gene expression and HBMSC differentiation towards the valvular phenotype is narrow. In addition, a specific OSI for stem-cell derived tissue engineering experiments using pulsatile flow mechanical conditioning was quantified here using a series of CFD simulations.

Moreover, use of an OSI value $(\mathrm{OSI}=0.23)$ taken from the sweet-spot OSI range $(0.18$ $\leq \mathrm{OSI} \leq 0.23)$ resulted in preferential valvular gene expression and phenotype in HBMSC culture experiments that were conducted in the current study. Thus, the OSI "sweet-spot" can now serve as a target for construct optimization for functional heart valve repair and/or replacement regenerative therapies.

\section{SPECIFIC AIM 2}

Aortic valve calcification is a predominant cause for aortic insufficiency [43, 18, 40]. While there are surgical treatment options available, they have distinct limitations and are generally performed when the native valve is already beyond repair. Additionally, pharmacological management of calcific valve disease during early stages is currently unavailable. Therapeutic discoveries in the treatment of early stages of aortic valve calcification requires a fundamental understanding of cellular mechanisms that are regulated by both the local biochemical and biomechanical environments and have been the subject of extensive research.

From a biomechanics standpoint, fluid-induced shear stresses across the leaflets have been closely linked to normal and pathological valve tissue remodeling activity $[41,14$, $8,10,17,16,44]$, but specific shear stress patterns and magnitudes both spatial and 
temporal have not yet been strongly linked to the underlying cell autocrine and paracrine signaling events that regulate the valve extracellular matrix (ECM). Nonetheless, the incidence of low shear, disturbed laminar flow resulting in temporal blood flow oscillatory patterns on the fibrosa side of aortic valve leaflets has been shown to strongly correlate to regions of calcific nodule deposition and aggregation $[10,17]$. The resulting oscillatory shear stresses (OSS) have thus been implicated in valve pathology [41, 45]. We have previously shown that OSS promotes the formation of engineered heart valve tissues $[5,6,12]$. Collectively, the common theme in valve tissue engineering and calcification in the context of OSS is its association with active tissue remodeling activity. However, the distinction between these scenarios may ultimately lie in the specific magnitude of oscillations that trigger the synthesis of healthy versus pathological valve ECM. Even less understood is the regional changes in fibrosa OSS that occur with aging.

As a first step in elucidating connections between OSS and calcification on the aortic valve surfaces, here, we took a computational approach to determine changes in OSS patterns on the human aortic valve fibrosa before and after calcific nodule deposition. We subsequently compared these findings to regions of the fibrosa that did not develop any pathology.

\subsection{Methodology for Specific Aim 2}

All simulations in this study were carried out using commercially available software (ANSYS® Workbench 2016, Ansys Inc., Canonsburg, PA) within a Windows ${ }^{\circledR} 7$ 64-bit 
Operating System environment (Microsoft Inc., Redmond, WA). A workstation with dual processors was used to facilitate an efficient and accurate solution convergence (Intel Xeon® X5550, 2.66 GHz processor speed, Intel Inc., Santa Clara, CA).

Computed tomography (CT) reconstructions of a human aortic valve with and without severe calcification (Fig. 18a, b) were obtained (82-year old female; Valve-012 HeartPrint catalog, Materialise Inc., Plymount MI). The temporal position of the valve structure depicted the early-diastolic phase wherein the leaflets would be in the process of closing. The valve geometry without calcific nodules (fig. 18c) was first discretized utilizing a tetrahedral mesh density $\left(1.70 \times 10^{3}\right.$ nodes and $7.73 \times 10^{3}$ elements, fig. $\left.18 \mathrm{e}\right)$. Calcified nodules were then placed as spatially determined from CT images to the healthy valve geometry (fig. 18d); as before, a tetrahedral mesh $\left(4.89 \times 10^{4}\right.$ nodes, $2.63 \times 10^{5}$ elements, fig. $\left.18 f\right)$ was subsequently generated for the calcified valve.

The fluid was modelled as blood, using a viscosity and density of 3.5 centipoise and $1.06 \mathrm{~g} / \mathrm{cm}^{3}$, respectively. A no-slip boundary condition was applied to the entire geometry for each valve model. Pulsatile flow simulations were run for the healthy and diseased aortic valve with solution convergence interpreted to have occurred when residuals arising from the continuity and momentum equations were able to meet a convergence criterion of $1 \times 10^{-9}$. 


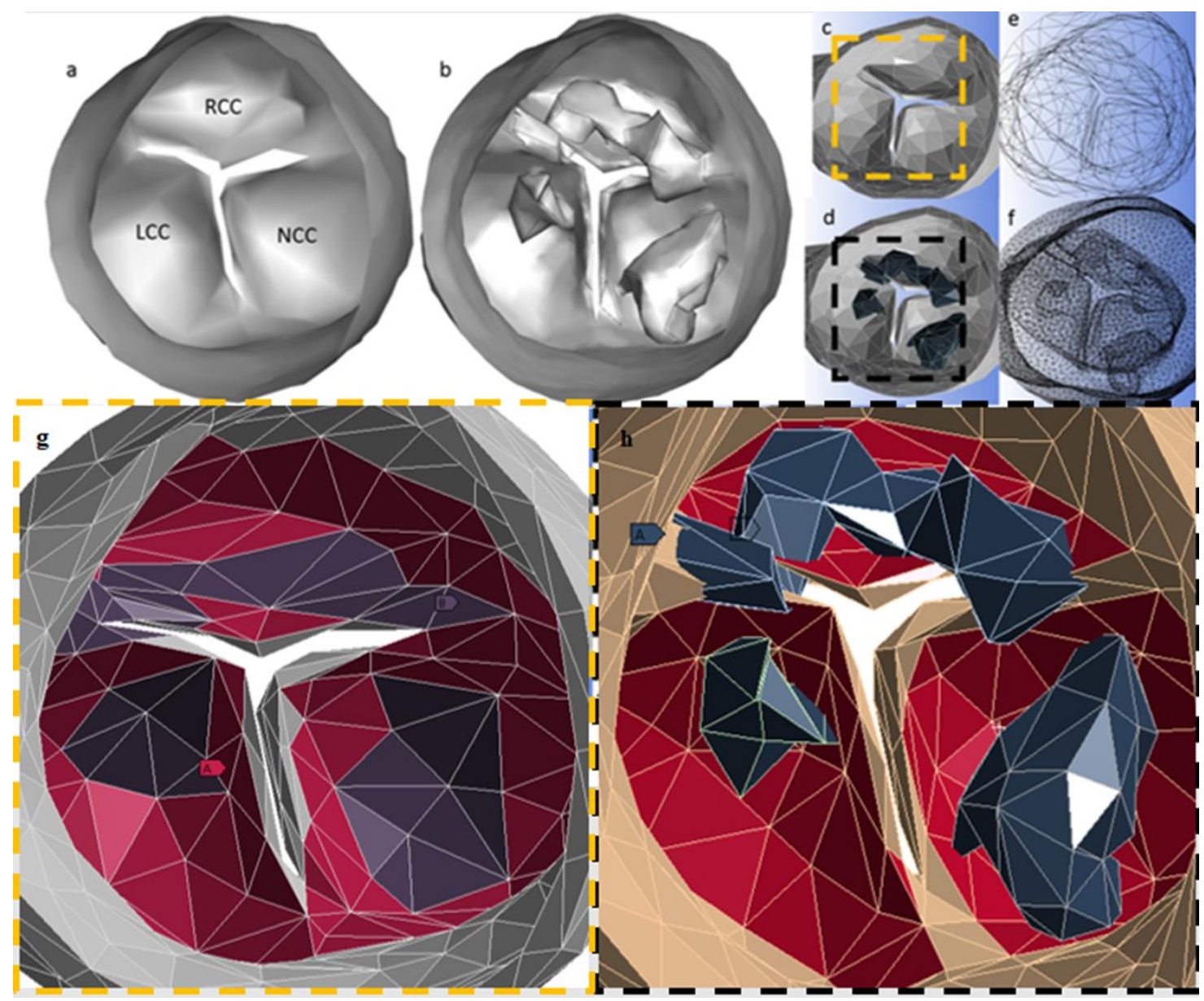

Figure 18: (a) Healthy aortic valve Fibrosa geometry in early diastole, with left coronary cusp (LCC), right coronary cusp (RCC) and non-coronary cusp (NCC), before and (b) after calcification. (c)The simulated healthy valve fibrosa model and (d) its corresponding mesh, had calcified nodules (black) added across the leaflets for (e) the diseased valve model and (f) its corresponding mesh. Areas of interest (A1, A2, A3, A4) on those regions represented both calcified areas (A3) and unaffected areas of the diseased valve (A4) and the areas corresponding to the calcified regions (A1) and unaffected regions (A2) on the healthy valve. (h) On the diseased valve, calcified deposits were analyzed both independently and as a collective zone (area A3, black) [A3-NCC $\left(126 \mathrm{~mm}^{2}\right.$, located at distal belly of NCC), A3-RCC (117.2 $\mathrm{mm}^{2}$, located at tips and proximal belly of RCC) and A3-LCC (51.9 $\mathrm{mm}^{2}$, located at proximal belly of LCC)] along with the regions surrounding the deposits (area A4, red) [A4NCC: $194.3 \mathrm{~mm}^{2}$; A4-RCC: $203.6 \mathrm{~mm}^{2}$; A4-LCC: $240 \mathrm{~mm}^{2}$ ] within each specific cusp. (g) The deposit regions corresponding to the diseased valve were then analyzed across the healthy valve (area A1, black) [A1-NCC: $144.93 \mathrm{~mm}^{2}$; A1-RCC: $81.08 \mathrm{~mm}^{2}$; A1-LCC:17.07 $\mathrm{mm}^{2}$ ] along with the areas consistent with the regions surrounding the deposits (area A2, red) [A2-NCC: $151.26 \mathrm{~mm}^{2}$; A2-RCC: $218.16 \mathrm{~mm}^{2}$; A2-LCC:291.24 $\mathrm{mm}^{2}$ ]. 


\subsubsection{Inlet and Outlet Boundary Conditions}

An aortic waveform used in our previous aim and originally described by Lotz et al. [36] was prescribed to the inlet while an $80 \mathrm{~mm} \mathrm{Hg}$-pressure boundary condition was prescribed to the outlet.

\subsubsection{Numerical Independence}

Independence testing on both valve geometries was carried out to optimize the computational conditions as follows:

\subsubsection{Mesh Independence}

For the healthy valve geometry, an initial, fine tetrahedral mesh density of $6.67 \times 10^{4}$ nodes and $3.62 \times 10^{5}$ elements was used. After running through a steady state simulation of 30 iterations, an optimized tetrahedral mesh density of $1.70 \times 10^{3}$ nodes and $7.73 \times 10^{3}$ elements was determined after finding the averaged wall shear stress of the valve and surrounding anatomy was within $5 \%$ of the initial fine mesh. Subsequently, the diseased valve was assigned an initial, fine tetrahedral mesh density of $1.21 \times 10^{5}$ nodes and $6.69 \times 10^{5}$ elements. An optimized tetrahedral mesh density of $4.89 \times 10^{4}$ nodes and $2.63 \times 10^{5}$ elements was determined, using a steady state simulation of 30 iterations, to find the averaged wall shear stress of the valve and surrounding anatomy, similarly was within $5 \%$ of its initial, fine mesh valve. 


\subsubsection{CFL-timestep selection and subsequent CFD model validation}

For both valve geometries utilized, the Courant-Friedrichs-Lewy condition was satisfied with a Courant number $<1$ for a timestep of $1 \mathrm{~ms}$ [46]. In addition, with the 80 mm Hg outlet condition satisfied, both healthy and diseased valve geometries exhibited a computed average inlet pressure of within $5 \%$ of a physiologically realistic value of $120 \mathrm{~mm} \mathrm{Hg}$.

\subsubsection{Cyclic Independence}

Three cycles were prescribed to the inlet of each valve geometry. The healthy valve model had an error of $2 \%$ between the $1^{\text {st }}$ and $3^{\text {rd }}$ cycles and $1.7 \%$ between the $2^{\text {nd }}$ and $3^{\text {rd }}$ cycle. The diseased valve had an error of $14.9 \%$ between the $1^{\text {st }}$ and $3^{\text {rd }}$ cycle

and $1.7 \%$ between the $2^{\text {nd }}$ and $3^{\text {rd }}$ cycle. Ultimately, the $3^{\text {rd }}$ cycle was selected for analysis in the healthy valve model and the diseased valve model.

\subsection{Results of Specific Aim 2}

\subsubsection{Time-Averaged Shear Stress}

The spatial distributions of axial time-averaged wall shear stresses (TAWSS) were presented on the healthy valve (Fig. 19a) and diseased valve (Fig. 19c).

\subsubsection{Healthy Valve Model}

The ventricularis side of the valve (fig. 20a) had an average ( \pm SEM) axial shear stress of $1.25 \pm 0.0195 \mathrm{dynes} / \mathrm{cm}^{2}$. As expected, the distribution of the shear stresses on the 
fibrosa side was relatively low in magnitude. The maximum axial shear stresses on the valve were concentrated at the leaflet tips while minimum, negative shear stresses were
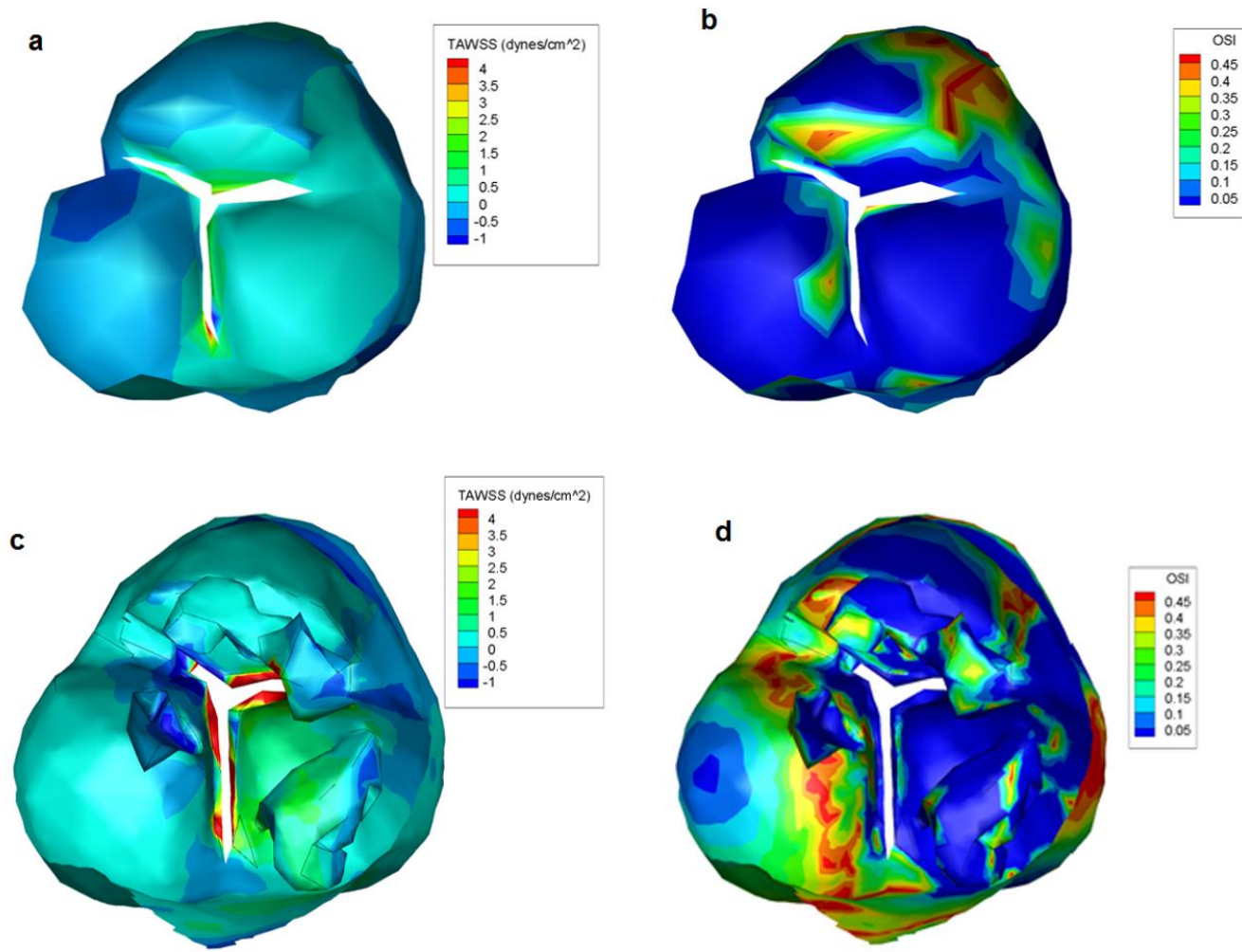

Figure 19: Healthy Valve spatial distribution of axial time-averaged wall shear stresses (a, mean: 0.091 dynes/cm2) and OSI (b, mean: 0.0998). Calcified Valve spatial distribution of axial time-averaged wall shear stresses (c, mean: 0.323 dynes $/ \mathrm{cm} 2)$ and OSI (d, mean: 0.1557). 

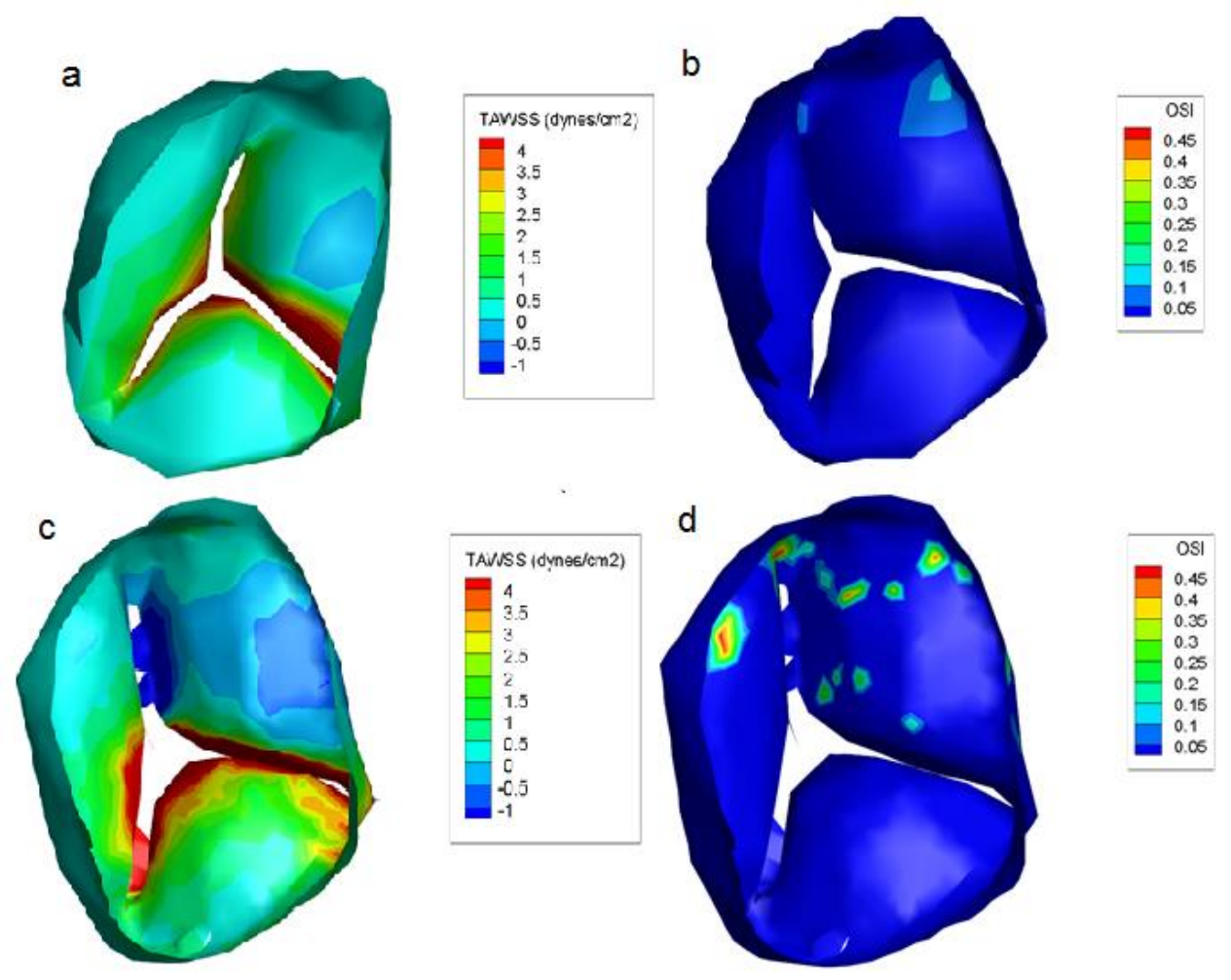

Fig. 20: Healthy ventricularis geometry plots of (a) axial time-averaged wall shear stress (TAWSS) (mean: 1.25 dynes $/ \mathrm{cm}^{2}$ ) and (b) OSI (mean: 0.003457) as well as diseased ventricularis geometry plots for (c) axial TAWSS (mean: 1.95 dynes $/ \mathrm{cm}^{2}$ ) and (d) OSI (mean: 0.01)

predominantly located across the fibrosa LCC. The mean fibrosa axial stress value of the valve was $0.091 \pm 0.067 \mathrm{dynes} / \mathrm{cm}^{2}$.

\subsubsection{Calcified Valve Model}

The ventricularis side of the valve (fig. 20c) exhibited an average axial shear stress average $\left( \pm\right.$ SEM) of $1.95 \pm 0.001$ dynes $/ \mathrm{cm}^{2}$. Maximum shear stresses were, as in the case of the healthy valve, located at the leaflet tips. Minimum, negative stresses were concentrated at the surface of the LCC (fig. 19c). The average fibrosa axial shear stress value of the valve was $0.32 \pm 0.004 \mathrm{dynes} / \mathrm{cm}^{2}$. 


\subsubsection{Oscillatory Shear Index}

The spatial distributions of OSI were computed for the healthy valve (Fig. 19b) and diseased valve (Fig. 19d) cases; averaged OSI values were subsequently summarized (Table 3, Table 4). OSI of Cusp-designated regions A1 - A4 (Fig. 18g-18h) were also reported (Table 3) with the following observations:

\subsubsection{Healthy Valve Model}

OSI on the ventricularis side of the valve was negligible (fig. 20b, mean OSI $=0.004$ ). On the other hand, on the fibrosa side, the OSI was relatively much higher on the RCC $(\mathrm{OSI}=0.147)$ while the NCC had an averaged OSI of 0.060 and the LCC had an OSI of 0.027. The OSI average of the entire fibrosa side was computed to be OSI $=0.103$.

\subsubsection{Calcified Valve Model}

Similar to the healthy valve, the OSI on the ventricularis side of the valve was substantially lower than that of the fibrosa (fig. 20d, mean OSI $=0.010$ ). The OSI field across the LCC (plaque area: 5.23\%; mean OSI =0.211; Table 2) had a distinct, nonuniform distribution with high OSI regions surrounding the calcified deposit on its surface (fig. 19d). Additionally, the RCC (plaque area: 39.8\%) and the NCC (plaque area: $38.2 \%$ ) exhibited a mean OSI of 0.131 and 0.051 , respectively. The OSI of the entire fibrosa side was computed to be 0.156 . 


\subsubsection{Regions that developed calcification}

Regions that developed calcification, A3 on the NCC, RCC and LCC, had mean OSIs of $0.06,0.101$, and 0.108 respectively with the collective OSI of these regions (area A3, Fig. 18h) exhibiting an overall Mean \pm SEM OSI: $0.089 \pm 0.015$. The corresponding regions of $\mathrm{A} 3$ on the healthy valve were $0.009,0.232$, and 0.023 with the mean OSI of these regions (area A1, Fig. 18g): $0.088 \pm 0.0721$. The unaffected regions surrounding A3 on the NCC, RCC and LCC had OSIs of 0.0267, 0.1522, and 0.2411, respectively, with the mean OSI of these regions (area A4, Fig. 18h): $0.14 \pm 0.0622$. Corresponding regions for A4 on the healthy valve had OSIs of $0.05,0.215$, and 0.044 with a mean OSI of these regions (area A2, Fig. 18g): $0.103 \pm 0.056$.

\subsection{3. \% Change in OSI}

The percent decrease in OSI (Table 4) in the region of the calcified valve (A3) compared to the same location when it was not diseased (A1) was found to be marginal $(1.59 \%)$. On the other hand, the regions that remained unaffected by calcification on both valves (A2, A4) had a much more substantial decrease in OSI by $35.9 \%$. 


\begin{tabular}{|l|l|l|l|}
\hline Aortic Valve Side & Healthy OSI & Diseased OSI & $\begin{array}{l}\text { Plaque } \\
\text { Percentage }\end{array}$ \\
\hline Ventricularis & 0.0035 & 0.01 & $0 \%$ \\
\hline NCC & 0.0603 & 0.0393 & $38.2 \%$ \\
\hline LCC & 0.0268 & 0.2114 & $5.23 \%$ \\
\hline RCC & 0.1472 & 0.1310 & $39.8 \%$ \\
\hline
\end{tabular}

Table 2: Healthy and diseased OSI on both ventricularis and fibrosa sides of valve with percent of calcified plaque occupying each cusp. Particularly, there was a significant change in OSI between the diseased and healthy LCC $(\mathrm{p}<0.05)$.

\begin{tabular}{|l|l|l|l|l|}
\hline Calcified Region & A1 & A2 & A3 & A4 \\
\hline NCC & 0.009 & 0.05 & 0.0597 & 0.0267 \\
\hline LCC & 0.232 & 0.215 & 0.1009 & 0.1522 \\
\hline RCC & 0.023 & 0.044 & 0.1077 & 0.2411 \\
\hline Mean \pm SEM & $0.088 \pm 0.0721$ & $0.103 \pm 0.056$ & $0.0894 \pm 0.015$ & $\begin{array}{l}0.14 \pm \\
0.0622\end{array}$ \\
\hline
\end{tabular}

Table 3: OSI on cusp-designated areas of valve fibrosa (A1-A4) for each region. Note that A3 and A4 are the calcified regions and unaffected regions of the calcified valve, respectively, while A1 and A2 are the corresponding calcified regions and corresponding unaffected regions on the healthy valve. Mean \pm SEM is provided for each area. A slight shift in OSI (1.6\%) occurred between the means of $\mathrm{A} 1$ and $\mathrm{A} 3$.

\begin{tabular}{|l|l|l|l|l|}
\hline $\begin{array}{l}\text { \%Change } \\
\text { (Mean A1 } \\
\text { Mean A3) }\end{array}$ & $\begin{array}{l}\text { \%Change } \\
\text { (Mean A2 \& } \\
\text { Mean A4) }\end{array}$ & $\begin{array}{l}\text { \% Change of } \\
\text { mean OSI in } \\
\text { NCC }\end{array}$ & $\begin{array}{l}\text { \% Change of } \\
\text { mean OSI in } \\
\text { RCC }\end{array}$ & $\begin{array}{l}\text { \%Change } \\
\text { of mean } \\
\text { OSI in } \\
\text { LCC }\end{array}$ \\
\hline $\begin{array}{l}1.59 \text { (SD: } 1.12, \\
\text { SEM:0.80) }\end{array}$ & $\begin{array}{l}35.9 \text { (SD: } 25.4, \\
\text { SEM:18) }\end{array}$ & 34.8 & 11.0 & 688.0 \\
\hline
\end{tabular}

Table 4: Percent (\%) change in mean OSI of cusp-designated regions and in mean OSI across distinct valvular cusps. There was a markedly low percent change between the means of A1 and A3 (1.6\%) compared to the change between the means of A2 and A4 (36\%). 


\subsection{Discussion of Specific Aim 2}

Axial TAWSS results from both affected and unaffected regions of both valve models (Fig. 19a, 19c) revealed non-unidirectional flow existed across the fibrosa, demonstrating that the native valve environments could produce OSS. The computed shear stresses of the aortic valve model simulated here had values [-5.94 dynes $/ \mathrm{cm}^{2}-$ 54.7 dynes $\left./ \mathrm{cm}^{2}\right]$ within the ranges previously reported in the literature $[11,30,17,16]$ with relatively lower shear stresses found on the fibrosa side (mean: $0.091 \mathrm{dynes} / \mathrm{cm}^{2}$ ) compared to the ventricularis side of each model (mean:1.25 dynes $/ \mathrm{cm}^{2}$ ).

The aortic valve OSI environment shifted remarkably between healthy and diseased valve physiologies. Consistent with previous work [48, 47, 17], our findings demonstrated that the NCC and RCC were the most susceptible to calcification. The healthy valve OSI for the NCC (OSI mean: 0.060), RCC (mean: 0.131) and LCC (mean: 0.027) established that OSI across a healthy valve geometry could vary between the three aortic valve leaflets; the diseased valve OSI of the NCC (OSI mean: 0.039), RCC (mean: 0.147) and LCC (mean: 0.211) similarly also varied. These findings suggest that relative changes in OSI or lack thereof between healthy and valve pathologies could potentially be used to predict regions on the valve fibrosa prone to calcification. Specifically, we speculate that in situations where the rate of valve matrix remodeling slows down, a change in OSI is unlikely, in time, making the valve susceptible to triggering abnormal ECM synthesis. The current study was able to confirm that indeed, regions susceptible to high calcification had only marginal changes in the OSI (healthy tissue area, A1: Mean \pm SEM: $0.088 \pm 0.072$ and same tissue area A1, after calcification, 
i.e., A3: Mean \pm SEM: $0.089 \pm 0.015$ ), resulting in a $\%$ change in OSI of only $1.59 \%$ ).

Conversely, regions of the aortic valve that did not get calcified, i.e. A2 and later, A4, exhibited a rather substantial \% change in OSI of nearly $36 \%$. We interpret that regional ECM remodeling of the fibrosa, particularly in the primary ECM components of the valve, i.e., collagen, glycosaminoglycans, and elastin, alter the valve shape which will change the OSI as would be expected in the localized leaflet surface environment. However, when this remodeling does not occur or occurs slower than needed, the OSI does not change, potentially triggering a disease pathway. In particular, since aortic valve calcification is strongly correlated with the aging process, it is likely that the slow turnover of elastin remodeling in the valve may be primarily associated with changes in OSI that occurs over decades, and leads to pathologies later in life, as recent studies have similarly suggested $[53,50,54,8,49,51,11,43,52,10]$

The findings in this brief report are very preliminary and contain two major limitations in the approaches. Firstly, a single patient's valve was analyzed before and after calcification. Secondly, the flow physics in a pulsatile flow model without accounting for valve leaflet motion was simulated during the early-diastolic, closing phase of the valve. While the absolute OSI values would be very different from those uncovered here, the general trend that negligible longitudinal changes in OSI co-relate with a higher probability of disease due to insufficient valve ECM remodeling, is likely to hold. Thus, in conclusion, we present in these short communications, a new interpretation of OSI in the context of valve tissue remodeling. In cases of tissue regeneration, a set value of OSI may be targeted to promote de novo tissue growth in- 
vitro as we previously described in aim 1 . However, when the OSI remains relatively unchanged in the native aortic valve fibrosa environment, this may be suggestive of an absence of or insufficient valve ECM remodeling, which could lead to a triggering point for valve pathologies. Therefore, in addition to the OSI environment, a change in the longitudinal and regional leaflet, fibrosa-side OSI conditions with aging, or lack thereof may serve useful as a bio-marker (e.g. a \% change in OSI computed every 5 years after the age of 40) for the early detection of aortic valve calcification.

\section{CONCLUSION}

Through utilization of a computational approach, the results included in this thesis have demonstrated the application and significance of oscillatory flow, namely OSS, within heart valve tissue engineering and heart valve disease. Specifically, these results have shown that physiologically relevant OSI environments can produce an optimized phenotype for BMSC-derived engineered valve tissue. Pulsatile conditions that produce an OSI within the "sweet spot" range $(0.18 \leq \mathrm{OSI} \leq 0.23)$ can now be used in bioreactor designs for proper conditioning of TEHVs. Furthermore, results have additionally established the method of using changes in OSI in detecting aortic valve remodeling that predispose native valve tissue towards higher probabilities of leaflet calcification.

Current efforts are focused on in-vitro experiments that will provide quantifiable data to further support our findings. 


\section{REFERENCES}

[1] Dohmen, P. M., "Clinical results of implanted tissue engineered heart valves.," HSR Proc. Intensive Care Cardiovasc. Anesth., vol. 4, no. 4, pp. 225-31, 2012.

[2] Hasan, A. et al., "Biomechanical properties of native and tissue engineered heart valve constructs," J. Biomech., vol. 47, no. 9, pp. 1949-1963, Jun. 2014.

[3] Head, S. J., M. Çelik, and A. P. Kappetein, "Mechanical versus bioprosthetic aortic valve replacement," Eur. Heart J., vol. 38, no. 28, pp. 2183-2191, Jul. 2017.

[4] Cheung, D. Y., B. Duan, and J. T. Butcher, "Current progress in tissue engineering of heart valves: multiscale problems, multiscale solutions," Expert Opin. Biol. Ther., vol. 15, no. 8, pp. 1155-1172, Aug. 2015.

[5] Rath, S., M. Salinas, A. G. Villegas, and S. Ramaswamy, "Differentiation and Distribution of Marrow Stem Cells in Flex-Flow Environments Demonstrate Support of the Valvular Phenotype," PLoS One, vol. 10, no. 11, p. e0141802, Nov. 2015.

[6] Salinas, M. and S. Ramaswamy, "Computational simulations predict a key role for oscillatory fluid shear stress in de novo valvular tissue formation," $J$. Biomech., vol. 47, no. 14, pp. 3517-3523, Nov. 2014.

[7] Vermot, J. et al., "Reversing Blood Flows Act through klf2a to Ensure Normal Valvulogenesis in the Developing Heart," PLoS Biol., vol. 7, no. 11, p. e1000246, Nov. 2009.

[8] Ge, L. and F. Sotiropoulos, "Direction and Magnitude of Blood Flow Shear Stresses on the Leaflets of Aortic Valves: Is There a Link With Valve Calcification?," J. Biomech. Eng., vol. 132, no. 1, p. 14505, Jan. 2010.

[9] Chandra, S., N. M. Rajamannan, and P. Sucosky, "Computational assessment of bicuspid aortic valve wall-shear stress: implications for calcific aortic valve disease," Biomech. Model. Mechanobiol., vol. 11, no. 7, pp. 1085-1096, Sep. 2012.

[10] Sun, L., N. M. Rajamannan, and P. Sucosky, "Defining the role of fluid shear stress in the expression of early signaling markers for calcific aortic valve disease.," PLoS One, vol. 8, no. 12, p. e84433, 2013.

[11] Mongkoldhumrongkul, N., N. Latif, M. H. Yacoub, and A. H. Chester, "Effect of Side-Specific Valvular Shear Stress on the Content of Extracellular Matrix in Aortic Valves," Cardiovasc. Eng. Technol., Oct. 2016. 
[12] Salinas, M., S. Rath, A. Villegas, V. Unnikrishnan, and S. Ramaswamy, "Relative Effects of Fluid Oscillations and Nutrient Transport in the In Vitro Growth of Valvular Tissues," Cardiovasc. Eng. Technol., vol. 7, no. 2, pp. 170-181, Jun. 2016.

[13] Salinas, M., D. E. Schmidt, M. Libera, R. R. Lange, and S. Ramaswamy, "Oscillatory shear stress created by fluid pulsatility versus flexed specimen configurations," Comput. Methods Biomech. Biomed. Engin., vol. 17, no. 7, pp. 728-739, May 2014.

[14] Engelmayr, G. C., V. L. Sales, J. E. Mayer, and M. S. Sacks, "Cyclic flexure and laminar flow synergistically accelerate mesenchymal stem cell-mediated engineered tissue formation: Implications for engineered heart valve tissues," Biomaterials, vol. 27, no. 36, pp. 6083-6095, Dec. 2006.

[15] Novaro, G. M. et al., "Clinical Factors, But Not C-Reactive Protein, Predict Progression of Calcific Aortic-Valve Disease," J. Am. Coll. Cardiol., vol. 50, no. 20, pp. 1992-1998, Nov. 2007.

[16] Yap, C. H., N. Saikrishnan, and A. P. Yoganathan, "Experimental measurement of dynamic fluid shear stress on the ventricular surface of the aortic valve leaflet," Biomech. Model. Mechanobiol., vol. 11, no. 1-2, pp. 231-244, Jan. 2012.

[17] Yap, C. H., N. Saikrishnan, G. Tamilselvan, and A. P. Yoganathan, "Experimental measurement of dynamic fluid shear stress on the aortic surface of the aortic valve leaflet," Biomech. Model. Mechanobiol., vol. 11, no. 1-2, pp. 171-182, Jan. 2012.

[18] Stewart, B. F. et al., "Clinical factors associated with calcific aortic valve disease. Cardiovascular Health Study.," J. Am. Coll. Cardiol., vol. 29, no. 3, pp. 630-4, Mar. 1997.

[19] Nkomo, V. T., J. M. Gardin, T. N. Skelton, J. S. Gottdiener, C. G. Scott, and M. Enriquez-Sarano, "Burden of valvular heart diseases: a population-based study.," Lancet (London, England), vol. 368, no. 9540, pp. 1005-11, Sep. 2006.

[20] Ku, D. N., D. P. Giddens, C. K. Zarins, and S. Glagov, "Pulsatile flow and atherosclerosis in the human carotid bifurcation. Positive correlation between plaque location and low oscillating shear stress.," Arteriosclerosis, vol. 5, no. 3, pp. 293-302, May 1985.

[21] Sucosky, P., K. Balachandran, A. Elhammali, H. Jo, and A. P. Yoganathan, "Altered shear stress stimulates upregulation of endothelial VCAM-1 and ICAM1 in a BMP-4- and TGF-beta1-dependent pathway.," Arterioscler. Thromb. Vasc. Biol., vol. 29, no. 2, pp. 254-60, Feb. 2009. 
[22] Rabkin, E., S. P. Hoerstrup, M. Aikawa, J. E. Mayer, and F. J. Schoen, "Evolution of cell phenotype and extracellular matrix in tissue-engineered heart valves during in-vitro maturation and in-vivo remodeling.," J. Heart Valve Dis., vol. 11, no. 3, p. 308-14; discussion 314, May 2002.

[23] Parvin Nejad, S., M. C. Blaser, J. P. Santerre, C. A. Caldarone, and C. A. Simmons, "Biomechanical conditioning of tissue engineered heart valves: Too much of a good thing?," Adv. Drug Deliv. Rev., vol. 96, pp. 161-175, Jan. 2016.

[24] Chambers, J. et al., "Standards for heart valve surgery in a "Heart Valve Centre of Excellence': Table 1,” Open Hear., vol. 2, no. 1, p. e000216, Jul. 2015.

[25] Ramaswamy, S., S. M. Boronyak, T. Le, A. Holmes, F. Sotiropoulos, and M. S. Sacks, "A Novel Bioreactor for Mechanobiological Studies of Engineered Heart Valve Tissue Formation Under Pulmonary Arterial Physiological Flow Conditions," J. Biomech. Eng., vol. 136, no. 12, p. 121009, Dec. 2014.

[26] Konertz, W. et al., "Hemodynamic characteristics of the Matrix P decellularized xenograft for pulmonary valve replacement during the Ross operation.," J. Heart Valve Dis., vol. 14, no. 1, pp. 78-81, Jan. 2005.

[27] Zhou, J., S. Hu, J. Ding, J. Xu, J. Shi, and N. Dong, "Tissue engineering of heart valves: PEGylation of decellularized porcine aortic valve as a scaffold for in vitro recellularization," Biomed. Eng. Online, vol. 12, no. 1, p. 87, Sep. 2013.

[28] Weber, B. et al., "Injectable living marrow stromal cell-based autologous tissue engineered heart valves: first experiences with a one-step intervention in primates," Eur. Heart J., vol. 32, no. 22, pp. 2830-2840, Nov. 2011.

[29] Syedain, Z. et al., "6-month aortic valve implantation of an off-the-shelf tissueengineered valve in sheep.," Biomaterials, vol. 73, pp. 175-84, Dec. 2015.

[30] Sacks, M. S. and A. P. Yoganathan, "Heart valve function: a biomechanical perspective.," Philos. Trans. R. Soc. Lond. B. Biol. Sci., vol. 362, no. 1484, pp. 1369-91, Aug. 2007.

[31] Schmidt, D. et al., "Minimally-Invasive Implantation of Living Tissue Engineered Heart Valves," J. Am. Coll. Cardiol., vol. 56, no. 6, pp. 510-520, Aug. 2010.

[32] Roman, B. L. and K. Pekkan, "Mechanotransduction in embryonic vascular development," Biomech. Model. Mechanobiol., vol. 11, no. 8, pp. 1149-1168, Nov. 2012. 
[33] Adamo, L. and G. García-Cardeña, "Directed Stem Cell Differentiation by Fluid Mechanical Forces," Antioxid. Redox Signal., vol. 15, no. 5, pp. 1463-1473, Sep. 2011.

[34] Li, Y. J. et al., "Oscillatory fluid flow affects human marrow stromal cell proliferation and differentiation," J. Orthop. Res., vol. 22, no. 6, pp. 1283-1289, Nov. 2004.

[35] Riddle, R. C., A. F. Taylor, D. C. Genetos, and H. J. Donahue, "MAP kinase and calcium signaling mediate fluid flow-induced human mesenchymal stem cell proliferation.," Am. J. Physiol. Cell Physiol., vol. 290, no. 3, pp. C776-84, Mar. 2006.

[36] Lotz, J., C. Meier, A. Leppert, and M. Galanski, “Cardiovascular Flow Measurement with Phase-Contrast MR Imaging: Basic Facts and Implementation," RadioGraphics, vol. 22, no. 3, pp. 651-671, May 2002.

[37] Heckel, E. et al., "Oscillatory Flow Modulates Mechanosensitive klf2a Expression through trpv4 and trpp2 during Heart Valve Development," Curr. Biol., vol. 25, pp. 1-8, 2015.

[38] Combs, M. D. and K. E. Yutzey, "Heart Valve Development," Circ. Res., vol. 105, no. 5, pp. 408-421, Aug. 2009.

[39] Hinton, R. B. and K. E. Yutzey, "Heart Valve Structure and Function in Development and Disease," Annu. Rev. Physiol., vol. 73, no. 1, pp. 29-46, Mar. 2011.

[40] Wirrig, E. E. and K. E. Yutzey, "Transcriptional regulation of heart valve development and disease.," Cardiovasc. Pathol., vol. 20, no. 3, pp. 162-7, 2011.

[41] Cao, K., M. BukaČ, and P. Sucosky, "Three-dimensional macro-scale assessment of regional and temporal wall shear stress characteristics on aortic valve leaflets," Comput. Methods Biomech. Biomed. Engin., vol. 19, no. 6, pp. 603-613, Apr. 2016.

[42] Ramaswamy, S. et al., "The role of organ level conditioning on the promotion of engineered heart valve tissue development in-vitro using mesenchymal stem cells," Biomaterials, vol. 31, no. 6, pp. 1114-1125, Feb. 2010.

[43] Peeters, F. E. C. M. et al., "Calcific aortic valve stenosis: hard disease in the heart," Eur. Heart J., Nov. 2017.

[44] Zhang, Z., L. Yuan, P. D. Lee, E. Jones, and J. R. Jones, "Modeling of time dependent localized flow shear stress and its impact on cellular growth within 
additive manufactured titanium implants," J. Biomed. Mater. Res. Part B Appl. Biomater., vol. 102, no. 8, pp. 1689-1699, Nov. 2014.

[45] Cao, K. and P. Sucosky, "Computational comparison of regional stress and deformation characteristics in tricuspid and bicuspid aortic valve leaflets," Int. $j$. numer. method. biomed. eng., vol. 33, no. 3, p. e02798, Mar. 2017.

[46] Wendell, D. C. et al., "Including aortic valve morphology in computational fluid dynamics simulations: initial findings and application to aortic coarctation.," Med. Eng. Phys., vol. 35, no. 6, pp. 723-35, Jun. 2013.

[47] Parks, M. and T. Cartlidge, "9 Assessment of aortic valve calcification using contrast-enhanced computed tomography," Heart, vol. 103, no. Suppl 4, pp. A3A3, May 2017.

[48] Cujec, B. and C. Pollick, "Isolated thickening of one aortic cusp: preferential thickening of the noncoronary cusp.," J. Am. Soc. Echocardiogr., vol. 1, no. 6, pp. 430-2, Nov. 1988.

[49] Lindman, B. R. et al., "Calcific aortic stenosis.," Nat. Rev. Dis. Prim., vol. 2, p. 16006, 2016.

[50] Balachandran, K., P. Sucosky, and A. P. Yoganathan, "Hemodynamics and mechanobiology of aortic valve inflammation and calcification.," Int. J. Inflam., vol. 2011, p. 263870, Jul. 2011.

[51] Merryman, W. D. and F. J. Schoen, "Mechanisms of calcification in aortic valve disease: role of mechanokinetics and mechanodynamics.," Curr. Cardiol. Rep., vol. 15 , no. 5, p. 355, May 2013.

[52] Rathan, S. et al., "Identification of side- and shear-dependent microRNAs regulating porcine aortic valve pathogenesis," Sci. Rep., vol. 6, no. 1, p. 25397, Jul. 2016.

[53] Arjunon, S., S. Rathan, H. Jo, and A. P. Yoganathan, "Aortic valve: mechanical environment and mechanobiology.," Ann. Biomed. Eng., vol. 41, no. 7, pp. 133146, Jul. 2013.

[54] Fernández Esmerats, J., J. Heath, and H. Jo, "Shear-Sensitive Genes in Aortic Valve Endothelium.," Antioxid. Redox Signal., vol. 25, no. 7, pp. 401-14, 2016. 